\title{
Phosphorylation-mediated EZH2 inactivation promotes drug resistance in multiple myeloma
}

\author{
Jiro Kikuchi,, ${ }^{1}$ Daisuke Koyama, ${ }^{1}$ Taeko Wada, ${ }^{1}$ Tohru Izumi, ${ }^{2}$ Peter O. Hofgaard, ${ }^{3}$ Bjarne Bogen, ${ }^{3,4}$ and Yusuke Furukawa ${ }^{1}$ \\ 'Division of Stem Cell Regulation, Center for Molecular Medicine, Jichi Medical University, Shimotsuke, Tochigi, Japan. ²Division of Hematology, Tochigi Cancer Center, Utsunomiya, Tochigi, Japan. \\ ${ }^{3} \mathrm{KC}$ Jebsen Centre for Research on Influenza Vaccines, Institute of Immunology, and ${ }^{2}$ Department of Immunology, Centre for Immune Regulation, Oslo University Hospital and University of Oslo, Oslo, Norway.
}

\begin{abstract}
Alterations in chromatin modifications, such as histone methylation, have been suggested as mediating chemotherapy resistance in several cancer types; therefore, elucidation of the epigenetic mechanisms that underlie drug resistance may greatly contribute to the advancement of cancer therapies. In the present study, we identified histone H3-lysine 27 (H3K27) as a critical residue for epigenetic modification in multiple myeloma. We determined that abrogation of drug-induced H3K27 hypermethylation is associated with cell adhesion-mediated drug resistance (CAM-DR), which is the most important form of drug resistance, using a coculture system to evaluate stroma cell adhesion-dependent alterations in multiple myeloma cells. Cell adhesion counteracted anticancer drug-induced hypermethylation of H3K27 via inactivating phosphorylation of the transcription regulator EZH2 at serine 21, leading to the sustained expression of antiapoptotic genes, including ICF1, B cell CLL/lymphoma 2 (BCL2), and hypoxia inducible factor 1, $\alpha$ subunit (HIF1A). Pharmacological and genetic inhibition of the ICF-1R/PI3K/AKT pathway reversed CAM-DR by promoting EZH2 dephosphorylation and H3K27 hypermethylation both in vitro and in refractory murine myeloma models. Together, our findings identify and characterize an epigenetic mechanism that underlies CAM-DR and suggest that kinase inhibitors to counteract EZH2 phosphorylation should be included in combination chemotherapy to increase therapeutic index.
\end{abstract}

\section{Introduction}

Multiple myeloma (MM) is one of the most intractable malignancies characterized by the infiltration and growth of malignant plasma cells in the bone marrow (BM). MM cells are much less sensitive to chemotherapeutic agents in the $\mathrm{BM}$ microenvironment, depending on the interaction with BM stromal cells (BMSCs) (1). This type of drug resistance, termed cell adhesion-mediated drug resistance (CAM-DR), is a major obstacle to achieving cure in $\mathrm{MM}$ patients (2). Elucidation of the mechanisms underlying CAM-DR would greatly improve the treatment outcome of MM patients and thus has been a subject of extensive investigation for years.

Chromatin is a highly ordered structure composed of DNA, histones, and other chromosomal proteins. Changes in the chromatin structure enable the rapid and reversible regulation of gene expression and subsequent events, including drug responses, without a heritable genetic alteration. This process, called epigenetic regulation, is mainly accomplished through posttranslational modifications of histone tails (3). Among core histones, histone $\mathrm{H} 3$ plays a pivotal role in chromatin organization; $\mathrm{H} 3$ methylation at the lysine 4 (H3K4) and 36 (H3K36) residues is generally associated with open chromatin structures, whereas the methylation of histone $\mathrm{H} 3 \mathrm{~K} 27$ and $\mathrm{H} 3 \mathrm{~K} 9$ represents a hallmark of condensed chromatin at silent loci (4). Alterations in these modifications underlie various biological events; however, the epigenetic mechanisms underlying drug resistance have not

Conflict of interest: The authors have declared that no conflict of interest exists. Submitted: December 4, 2014; Accepted: September 17, 2015.

Reference information: / Clin Invest. 2015;125(12):4375-4390. doi:10.1172/JCI80325. been fully elucidated, although some studies have suggested the involvement of an altered chromatin state by H3K4 demethylation in anticancer drug tolerance $(5,6)$.

Among the histone $\mathrm{H} 3$ modifications, trimethylation at lysine 27 (H3K27me3) is known to be a critical contributor to oncogenesis. For example, the SET domain-containing histone methyltransferase MMSET/KMT3G is overexpressed in an aggressive form of MM carrying $\mathrm{t}(4 ; 14)$, leading to altered chromatin states by inducing an increase in H3K36me2 and decrease in H3K27me3 $(7,8)$. The oncogenic function of MMSET is closely associated with its catalytic activity in MM (9-11), making it amenable to therapeutic interventions. A reduction in $\mathrm{H} 3 \mathrm{~K} 27 \mathrm{me} 3$ is also caused by misregulation and/or mutations in the H3K27 methyltransferase EZH2/KMT6 and the H3K27 demethylase KDM6B/JMJD3 in numerous cancers, which results in altered transcription programs underlying malignant transformation (4). Furthermore, somatic mutations in H3K27 have been observed in aggressive gliomas $(12,13)$. These findings indicate that H3K27 methylation is profoundly implicated in oncogenesis; however, relatively little is known about its specific role in MM biology, including CAM-DR.

Here, we propose an epigenetic mechanism of drug resistance and its application to treatment strategies to overcome CAM-DR in MM. We found that anticancer drug-induced H3K27 hypermethylation is specifically abrogated during the acquisition of CAM-DR by MM cells. Direct contact with BMSCs causes phosphorylation-mediated EZH2 inactivation and subsequent H3K27 hypomethylation, which sustains the expression of antiapoptotic genes to promote drug resistance in MM cells. Importantly, this process could be a target for therapeutic interventions, as demonstrated in refractory murine MM models in this study. 
A
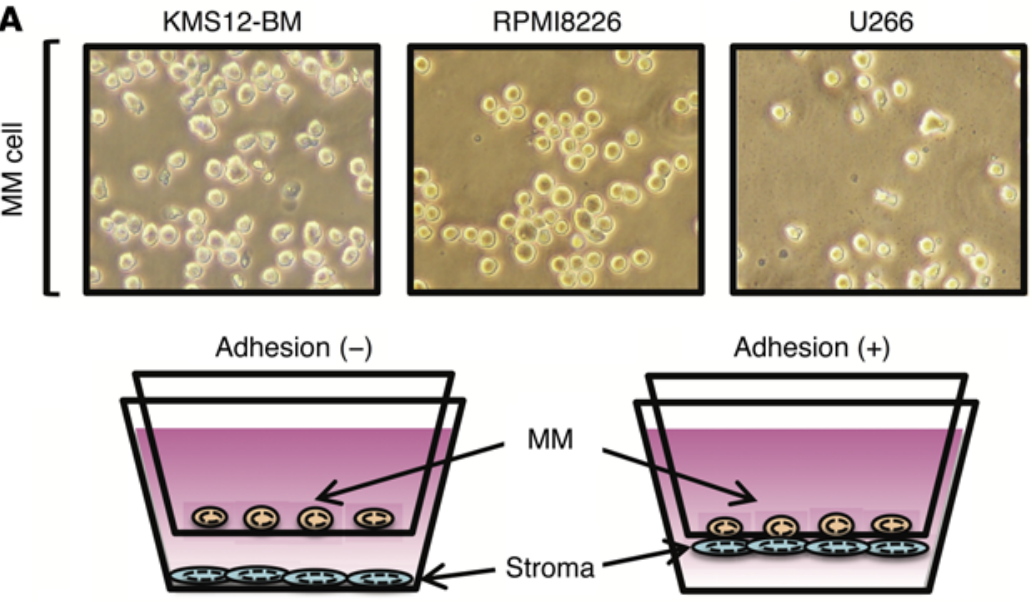

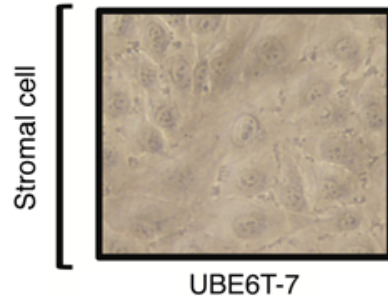

B

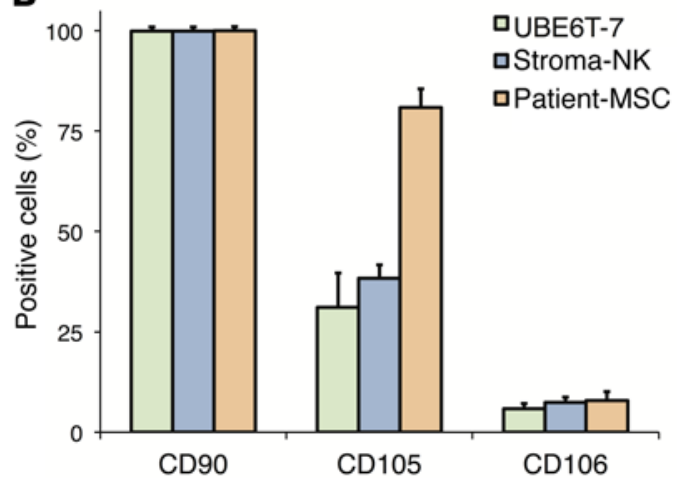

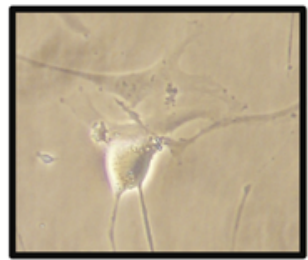

Patient-MSC

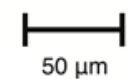

C

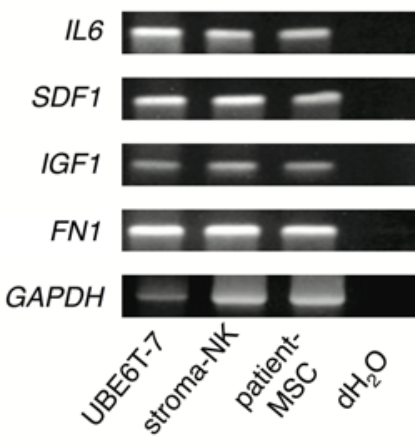

Figure 1. In vitro culture system to recapitulate CAM-DR. (A) Diagram of the culture system used in the present study. See Methods for a detailed description of the procedure. (B) Flow cytometric analysis of the expression of BMSC markers on UBE6T-7, stroma-NK, and MM patient-derived MSCs (patient-MSC). Means \pm SD of 3 independent experiments are shown. (C) Total cellular RNA was isolated from the indicated cells and subjected to semiquantitative RT-PCR analysis for the expression of IL6, SDF1, IGF1, FN1, and GAPDH (internal control), using primers listed in Supplemental Table 1. PCR without cDNA serves as a negative control $\left(\mathrm{dH}_{2} \mathrm{O}\right)$. The results of suboptimal amplification cycles (35 cycles) are shown. Detailed procedures are described in Supplemental Methods.

\section{Results}

Establishment of the in vitro culture system to recapitulate CAM$D R$. To investigate the epigenetic mechanisms underlying CAM-DR, we established the in vitro culture system using a cell-culture insert that allows coculture of MM cells and BMSCs with or without direct contact (Figure 1A and ref. 14). We used the human stromal cell lines UBE6T-7 and stroma-NK along with MM patient-derived mesenchymal stromal cells (MSCs) as BMSCs in this study. These cells expressed typical markers of BMSCs, such as CD90, CD105, IL6, SDF1, IGF1, and FN1, which represent key molecules for the induction of CAM-DR (Figure $1, B$ and C, ref. 15). Using this system, we investigated whether MM-BMSC interactions affected anti-MM drug-induced cytotoxicity in vitro. Direct adhesion to UBE6T-7 cells significantly reduced the cytotoxic activity of doxorubicin (ADM) and 4-hydroxycyclophosphamide (4-OHCY) in RPMI8226 and KMS12-BM cells (Figure 2A). Flow cytometric analyses revealed that direct contact significantly decreased the size of annexin V-positive and sub- $G_{1}$ fractions and increased the size of $\mathrm{S}$ and $\mathrm{G}_{2} / \mathrm{M}$ fractions in 4-OHCY-treated RPMI8226 cells (Supplemental Figure 1, A and B). These results indicate that our system could successfully reproduce CAM-DR at close to in vivo conditions and that it also serves as an ideal tool to elucidate its underlying mechanisms.

Cytotoxic drug-induced H3K27 hypermethylation is inhibited in cell adhesion-mediated drug-resistant MM cells. Using 
A
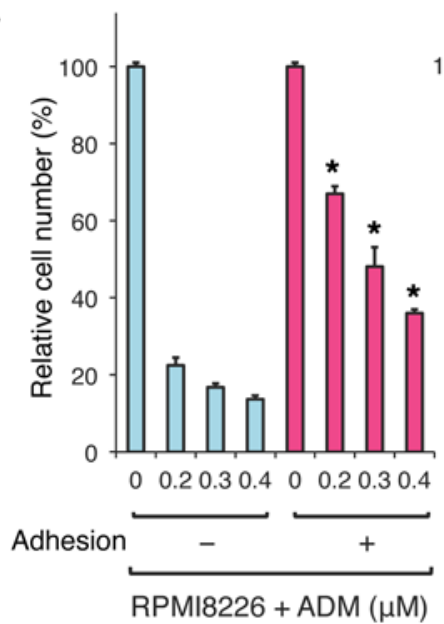

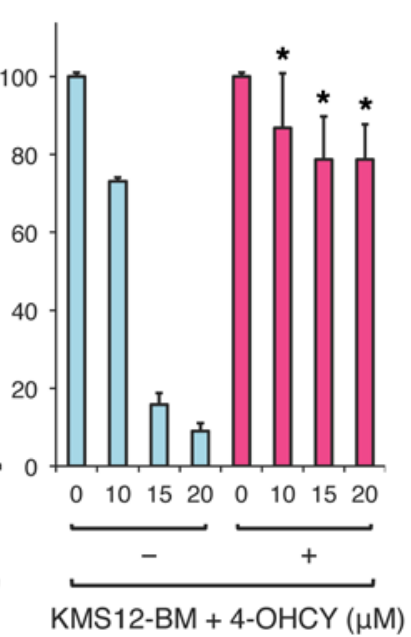

B

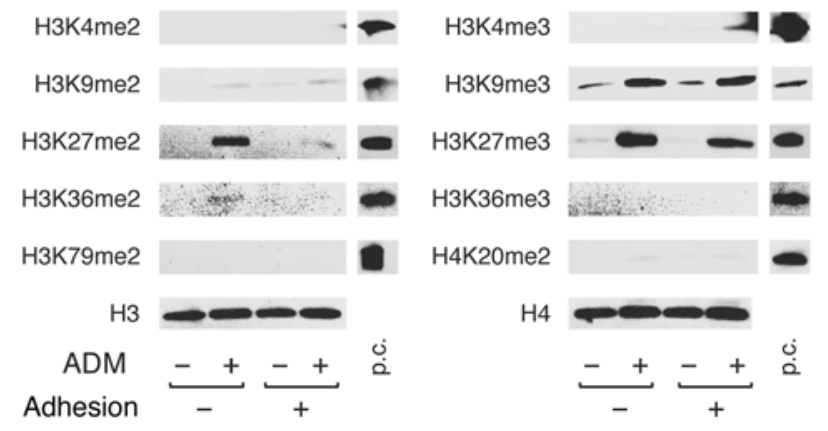

D

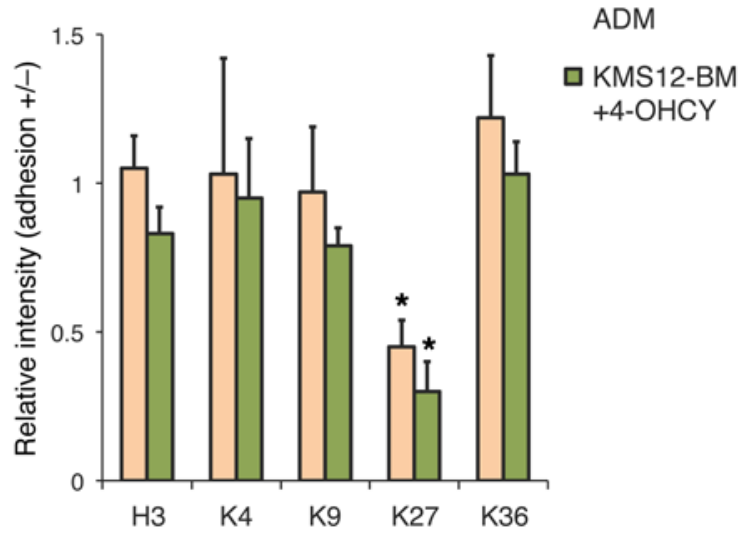

Figure 2. Cell adhesion confers drug resistance to MM cells by blocking drug-induced H3K27 hypermethylation in vitro. (A) RPMI8226 and KMS12-BM cells were cultured in the absence or presence of either ADM (left panel) or 4-OHCY (right panel) in a cell-culture insert with (+) or without (-) direct adhesion to UBE6T-7 cells. After 72 hours of culture, cell proliferation was assessed by the MTT reduction assay and expressed as a percentage of the values of corresponding untreated cells. ${ }^{*} P<0.05$ between adhesion $(-)$ and $(+)$ at the same drug concentration determined by 1-way ANOVA with the Student-Newman-Keuls multiple comparisons test $(n=3)$. (B) Nuclear extracts were prepared from RPMI8226 cells cultured in the presence of $0.4 \mu \mathrm{M}$ ADM for 48 hours and subjected to immunoblotting. We used recombinant histones $\mathrm{H} 3$ and $\mathrm{H} 4$ chemically modified at corresponding sites (Active Motif) as positive controls (p.c.). See Supplemental Figure 10 for antibody validation. (C) Whole cell lysates were prepared after 48 hours of culture described in $\mathbf{A}$ and subjected to immunoblotting. The signal intensities of each band were quantified, normalized to those of the corresponding histone $\mathrm{H} 3$, and shown as relative values setting untreated controls at 1.0. (D) Relative intensities of adhesion $(+) /(-)$ at doses of $0.4 \mu \mathrm{M} \mathrm{ADM}$ and $20 \mu \mathrm{M} 4-\mathrm{OHCY}$, respectively, in panel $\mathbf{C}$ are shown. ${ }^{*} P<0.05$ against the value of histone H3 calculated by 1 -way ANOVA with the Student-Newman-Keuls multiple comparisons test $(n=3)$.

the coculture system, we screened for global changes in histone methylation status during the acquisition of CAM-DR by MM cells. We isolated nuclear extracts during the experiments shown in Figure 2A and subjected them to immunoblot analysis for 10 different types of histone methylation whose biological significance is well understood (3). Among them, ADM increased the abundance of trimethylated H3K4 (H3K4me3), H3K9me3, H3K27me2/me3, and H3K36me2 in RPMI8226 cells under adhesion-free conditions (Figure 2B). This may reflect anticancer drug-induced changes in the cell-cycle distribution and concomitant histone methylation patterns (3). Next, we performed detailed studies of the 4 modifications using wholecell lysates from 2 cell lines treated with ADM and 4-OHCY. Anti-MM drugs reproducibly induced a moderate increase in the abundance of $\mathrm{H} 3 \mathrm{~K} 4 \mathrm{me} 3$, H3K9me3, and H3K36me2 irre- spective of adhesion to BMSCs (Figure 2C). Notably, H3K27me3 showed a completely different pattern. Without cell adhesion, anti-MM drugs markedly increased the abundance of H3K27me3 in a dose-dependent manner. This increase was significantly perturbed by direct adhesion to BMSCs in both RPMI8226 and KMS12-BM cells (Figure 2, C and D). The methylation status of H3K27 was inversely correlated with cell viability (compare Figure 2, A and C), whereas such correlation was not observed with other histone modifications. These results suggest that drug-induced increase in H3K27me3 is closely associated with efficacy and is abrogated during the acquisition of CAM-DR by MM cells. We then verified the correlation of H3K27 hypermethylation with drug sensitivity at individual cell levels by simultaneously detecting H3K27me3 and 7-aminoactinomycin D (7-AAD), a cell death marker, using flow cytometry. The propor- 
A

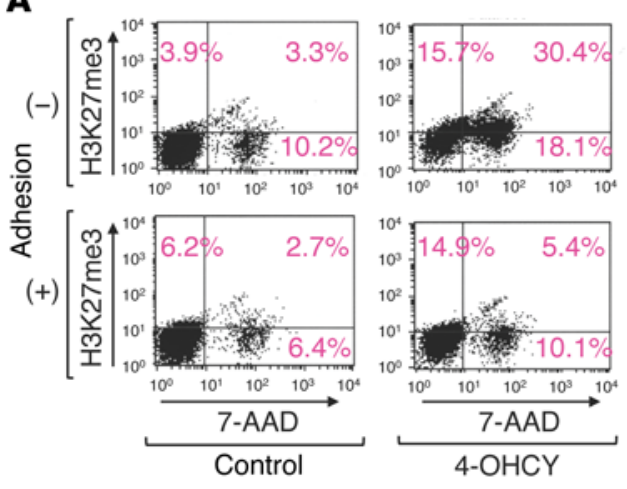

B

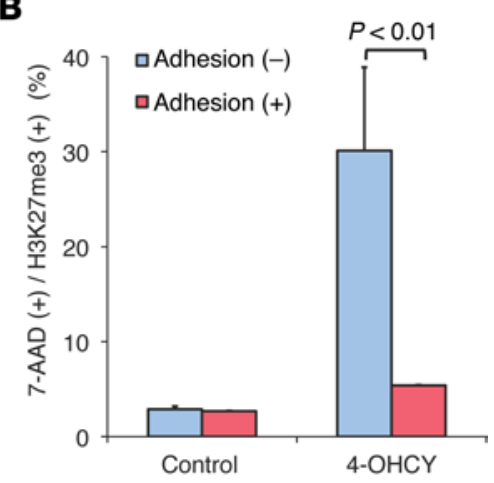

C

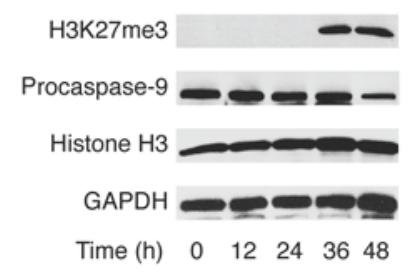

D

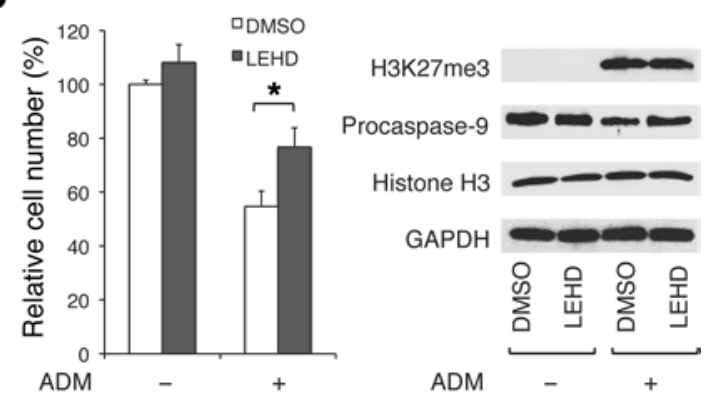

Figure 3. H3K27 hypermethylation is correlated with drug sensitivity but is not a consequence of cell death. (A) KMS12-BM cells were harvested after 48 hours of culture described in Figure 2A and stained with Alexa Fluor 488-conjugated anti-H3K27me3 and 7-AAD for flow cytometric analysis. (B) The $y$ axis shows the proportion of cells positive for both H3K27me3 and 7-AAD. $P$ values were calculated by 1-way ANOVA with the Student-Newman-Keuls multiple comparisons test $(n=3)$. (C) Whole cell lysates were prepared from RPMI8226 cells cultured with $0.4 \mu$ M ADM at the indicated time points and subjected to immunoblot analyses for H3K27 trimethylation and caspase-9 activation. (D) RPMI8226 cells were cultured in the absence (-) or presence (+) of $0.4 \mu \mathrm{M}$ ADM with or without a peptide inhibitor for caspase-9 (z-LEHD-fmk). Left panel: after 72 hours of culture, cell proliferation was determined by the MTT reduction assay and expressed as a percentage of the values of corresponding untreated cells. Mean \pm SD of 3 independent experiments are shown. $P$ values were calculated by 1 -way ANOVA with the Student-Newman-Keuls multiple comparisons test. ${ }^{*} P<0.05$. Right panel: whole cell lysates were prepared after 48 hours for immunoblotting.

tion of cells positive for both H3K27me3 and 7-AAD was markedly increased by 4-OHCY without cell adhesion. Direct contact to BMSCs selectively reduced the numbers of double-positive cells (Figure 3, A and B). Taken together, these data show that H3K27 hypermethylation reflects drug sensitivity in individual cells; high H3K27me3 cells are prone to die, and low H3K27me3 cells are resistant to drugs. However, it is possible that H3K27 hypermethylation is a bystander of chromatin condensation associated with apoptotic cell death (16). To negate this possibility, we compared the kinetics of H3K27 hypermethylation and caspase-9 activation during ADM-induced apoptosis. Immunoblot analyses revealed that H3K27 hypermethylation obviously preceded caspase- 9 activation, as determined by a decrease in the abundance of procaspase-9 (Figure 3C). Furthermore, a caspase-9 inhibitor did not impede H3K27 hypermethylation at a concentration that significantly suppressed ADM-mediated apoptosis (Figure 3D). These data indicate that H3K27 hypermethylation is not a simple consequence, but an upstream event of drug-induced apoptosis in MM.

Finally, we confirmed the above results using fibronectin and other BMSCs. Consistent with our previous reports in which adhesion-mediated signals from fibronectin-engaged VLA-4 play critical roles in the establishment of CAM-DR in MM cells $(17,18)$, the presence of fibronectin significantly attenuated the cytotoxic effects of ADM and dexamethasone in RPMI8226 and KMS12-BM cells (Supplemental Figure 2A). Fibronectin also abrogated druginduced increase in $\mathrm{H} 3 \mathrm{~K} 27 \mathrm{me} 3$, but not in $\mathrm{H} 3 \mathrm{~K} 4 \mathrm{me} 3, \mathrm{H} 3 \mathrm{~K} 9 \mathrm{me} 3$, or H3K36me2, in both cell lines (Supplemental Figure 2, B and C). Similarly, direct adhesion to stromal-NK cells and MM patientderived MSCs impaired ADM- and melphalan-induced (L-PAMinduced) H3K27 hypermethylation, which coincided with the induction of CAM-DR in RPMI2886 and U266 cells (Supplemental Figure 2, D-G).

Inhibition of $H 3 \mathrm{~K} 27 \mathrm{me} 3$ recapitulates $C A M-D R$ under stromafree conditions. Next, we investigated the effects of pharmacological modulation of histone $\mathrm{H} 3$ methylation on drug-induced cytotoxicity in MM cells under adhesion-free conditions. To achieve this goal, we used the inhibitors for H3K4me3-specific demethylase (2-PCPA), H3K27me3-specific demethylase (GSK-J4), H3K9me3-specific methyltransferase (chaetocin), and H3K27me3-specific methyltransferase (DZNep and GSK343) at suboptimal doses (Supplemental Table 2). Of these compounds, only H3K27me3 inhibitors, DZNep and GSK343, significantly ameliorated ADM- and 4-OHCY-induced cytotoxicity (Figure $4, \mathrm{~A}$ and $\mathrm{B}$ ) along with the obvious inhibition of $\mathrm{H} 3 \mathrm{~K} 27$ trimethylation (Figure $4 \mathrm{C}$ ) at 0.4 and $1.0 \mu \mathrm{M}$, respectively (cytotoxic 

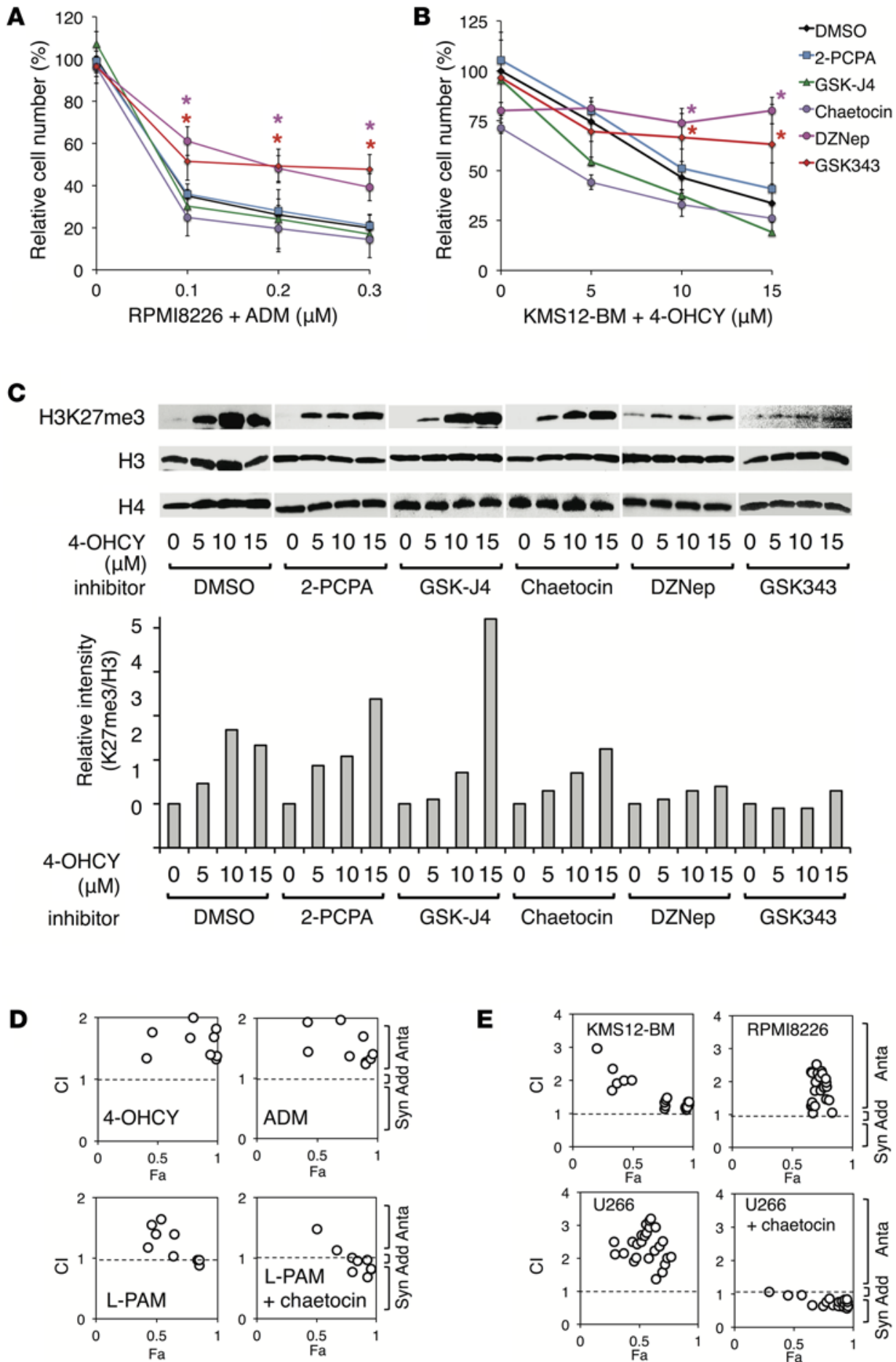

Figure 4. Pharmacological inhibition of H3K27me3 recapitulates CAM-DR under stroma-free conditions. RPMI8226 (A) and KMS12-BM (B) cells were cultured with $50 \mu \mathrm{M}$ 2-PCPA, $1 \mu \mathrm{M}$ GSK-J4, $0.03 \mu \mathrm{M}$ chaetocin (G9a inhibitor), $0.4 \mu \mathrm{M}$ DZNep (EZH2 inhibitor), $1.0 \mu \mathrm{M}$ GSK343 (EZH2 inhibitor), or vehicle alone (DMSO) for 72 hours in the absence or presence of either ADM or 4-OHCY. Cell viability was determined by the MTT reduction assay. ${ }^{*} P<0.05$ against the DMSO control determined by 1 -way ANOVA with the Student-Newman-Keuls multiple comparisons test $(n=3)$. (C) Upper panel: whole cell lysates were prepared after 48 hours of culture in the experiments described in B and subjected to immunoblotting. Lower panel: signal intensities of each band were quantified, normalized to those of the corresponding histone $\mathrm{H} 3$, and shown as relative values setting untreated controls at 1.0. (D) KMS12-BM cells were cultured with various concentrations of GSK343 in combination with 4-OHCY, ADM, or L-PAM and assessed for cell viability after 72 hours. Isobologram analysis was carried out using the CompuSyn software program to obtain the combination index (CI). The $\mathrm{Cl}$ values of less than 1.0, approximately 1.0, and greater than 1.0 indicate synergism (Syn), additive (Add), and antagonism (Anta), respectively, by definition. The same experiment was performed with a combination of L-PAM and chaetocin (L-PAM + chaetocin) as a control. (E) KMS12-BM, RPMI8226, and U266 cells were cultured with various concentrations of DZNep in combination with dexamethasone for the isobologram analysis. The same experiment was performed with chaetocin in U266 cells (U266 + chaetocin) as a control. 
A
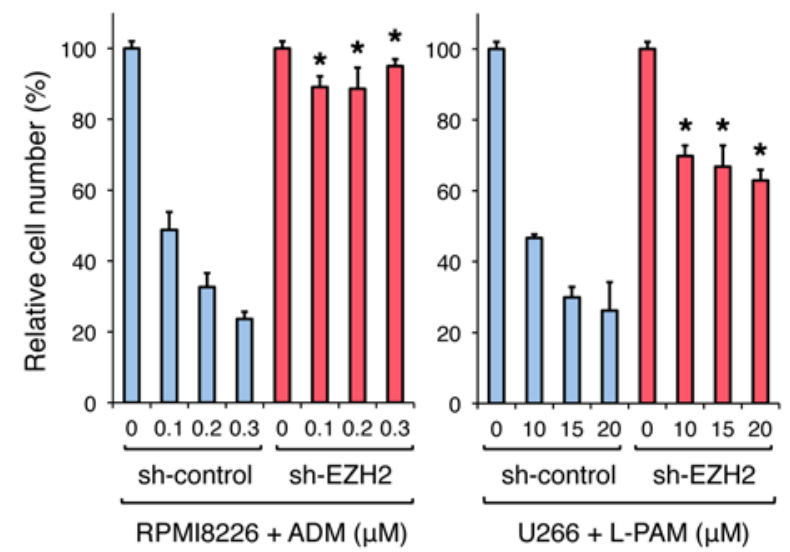

B

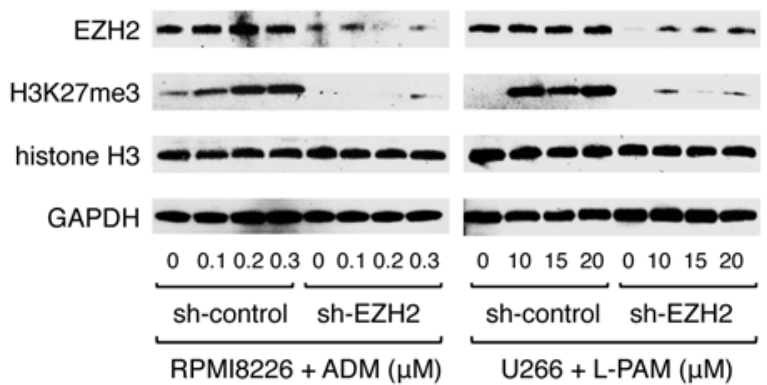

concentration $\sim 10 \mu \mathrm{M})$. An isobologram analysis of drug interactions revealed that the combination of H3K27 inhibitors and 3 anti-MM drugs was antagonistic in all $3 \mathrm{MM}$ cell lines tested (Figure 4, D and E). In contrast, the H3K9me3 inhibitor chaetocin had synergistic cytotoxic effects in combination with L-PAM (Figure 4D) or dexamethasone (Figure 4E). This is compatible with previous studies showing that it was not DZNep, but chaetocin, that exerted significant cytotoxicity against MM cells (19, 20). Furthermore, we examined the effects of EZH2 knockdown on anti-MM drug-induced cytotoxicity because H3K27 trimethylation was mainly catalyzed by EZH2. As anticipated, shRNA-mediated EZH2 knockdown significantly ameliorated ADM- and L-PAM-induced cytotoxicity along with the marked reduction in H3K27me3 in both RPMI8226 and U266 cells under stroma-free conditions (Figure 5, A and B). These results underscore the importance of EZH2-mediated H3K27 trimethylation in drug sensitivity in MM cells.

Finally, we asked whether MMSET exerted drug resistance via the same mechanisms because MMSET overexpression downregulates $\mathrm{H} 3 \mathrm{~K} 27 \mathrm{me} 3$ and has been identified as a marker of worse prognosis in MM patients $(7,8)$. To this end, we overexpressed MMSET in t(4;14)-negative RPMI8226 cells and knocked it down in $\mathrm{t}(4 ; 14)$-positive KMS-11 cells. As anticipated, $M M S E T$ overexpression significantly mitigated ADM-induced cytotoxicity along with the marked reduction in $\mathrm{H} 3 \mathrm{~K} 27 \mathrm{me} 3$ and a robust increase in H3K36me2 (Supplemental Figure 3A). In contrast, MMSET knockdown moderately but significantly enhanced L-PAM-induced cytotoxicity along with an increase in H3K27me3 and a striking decrease in H3K36me2 (Supplemental Figure 3B). Taken together, these data show that the inhibition of H3K27me3 by small molecular compounds, EZH2
Figure 5. Inhibition of H3K27me3 by EZH2 knockdown recapitulates CAM-DR under stroma-free conditions. (A) RPMI8226 and U266 cells were transduced with either the pLL3.7-sh-control (sh-control) or pLL3.7sh-EZH2 (sh-EZH2) lentiviral vector and cultured with various concentrations of ADM (left panel) or L-PAM (right panel) for 72 hours. ${ }^{*} P<0.05$ against sh-control determined by 1 -way ANOVA with the Student-Newman-Keuls multiple comparisons test $(n=3)$. (B) Whole cell lysates were prepared during the experiments described in $\mathbf{A}$ and subjected to immunoblotting. See Supplemental Table 3 for target sequences.

knockdown, and MMSET overexpression confers strong drug resistance to MM cells, reproducing CAM-DR under stromafree conditions. Therefore, cell adhesion-mediated H3K27 hypomethylation appears to play a central role in CAM-DR.

Phosphorylation-mediated EZH2 inactivation underlies $\mathrm{H} 3 \mathrm{~K} 27$ hypomethylation during CAM-DR induction. Next, we investigated the mechanisms by which drug-induced H3K27 hypermethylation is suppressed during the development of CAM-DR. First, we examined the expression of histone H3K27 methyltransferases (EZH1, EZH2, and G9a) and demethylases (UTX and JMJD3). Although a modest increase in the expression of EZH1 and G9a was induced by ADM under adherent conditions, its level was not proportional to the changes in corresponding methylation sites in anti-MM drug-treated cells (Supplemental Figure 4A and Figure 6, A and B). These results rather suggest the involvement of posttranslational mechanisms in H3K27 hypermethylation in drug-treated MM cells. Previous studies indicated that the catalytic activity of EZH2 was regulated by phosphorylation at serine-21 (S21), threonine-350 (T350), and threonine-492 (T492) $(21,22)$. We therefore examined the phosphorylation status of EZH2 using phospho-specific antibodies against these sites during the acquisition of CAM-DR by MM cells. EZH2 phosphorylation at S21 was suppressed by anticancer drugs in dose- and time-dependent manners without cell adhesion, but was retained or rather increased in the presence of BMSCs (Figure 6, A and B). In contrast, the phosphorylation status of T492 was not affected by either cell adhesion or drug treatment. Data quantification confirmed a marked increase in S21 phosphorylation by adhesion to BMSCs and a significant decrease without adhesion in ADM-treated MM cells (Figure 6C). Importantly, the abundance of $\mathrm{H} 3 \mathrm{~K} 27 \mathrm{me} 3$ showed a strong inverse correlation with the phosphorylation level of S21, but not that of T492 (Figure 6, A and B). Similar results were obtained in RPMI8226 and KMS12-BM cells in contact with fibronectin (Supplemental Figure 4B). Immunofluorescent staining validated the cell adhesion-mediated upregulation of S21 phosphorylation at the single cell level (Figure 6D). We and others have reported that bortezomib can overcome CAM-DR of MM cells $(17,23)$. This prompted us to determine the effects of bortezomib on S21 phosphorylation of EZH2 and H3K27 hypermethylation. As anticipated, bortezomib was able to induce H3K27 hypermethylation by perturbing EZH2 phosphorylation even in the presence of stromal cells, which was associated with the reversal of CAM-DR (Supplemental Figure 4C). To further delineate the role of S21 phosphorylation in CAM-DR, we used a dominant-negative inactive mutant of EZH2 in which S21 was replaced with aspartic acid to mimic phosphorylation (24). As shown in Figure 6E, 
A

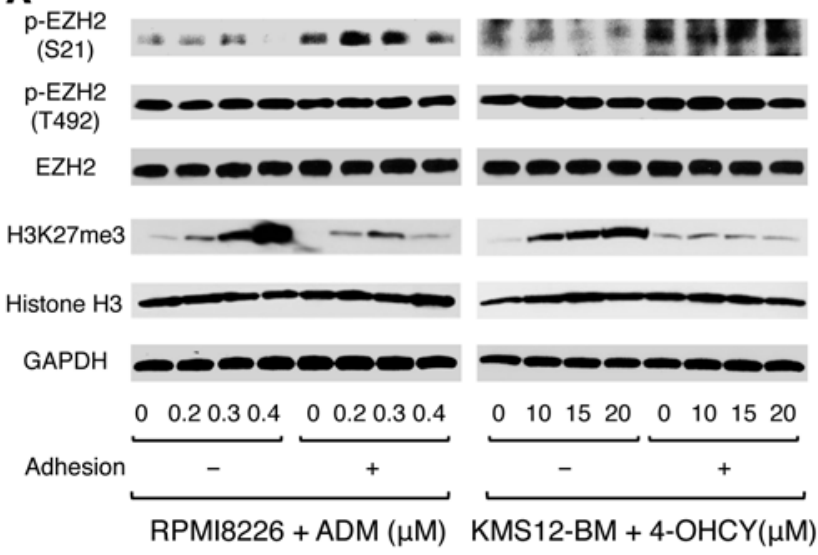

B

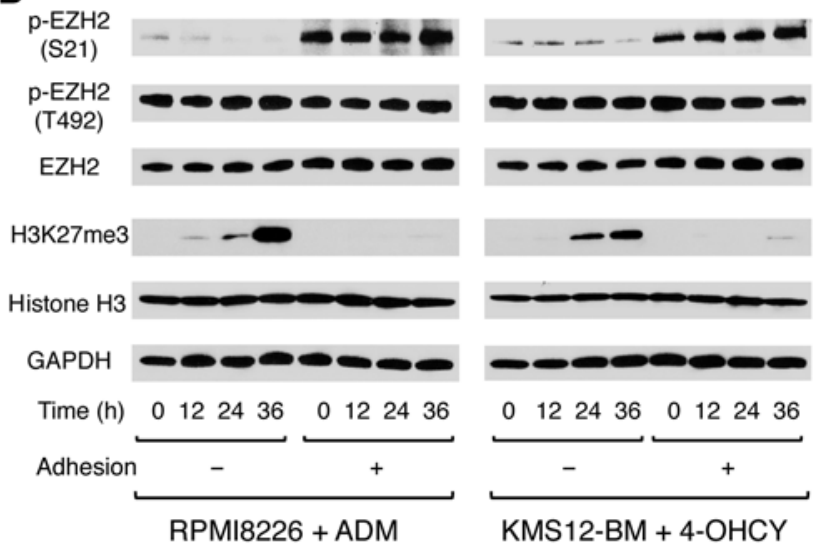

C

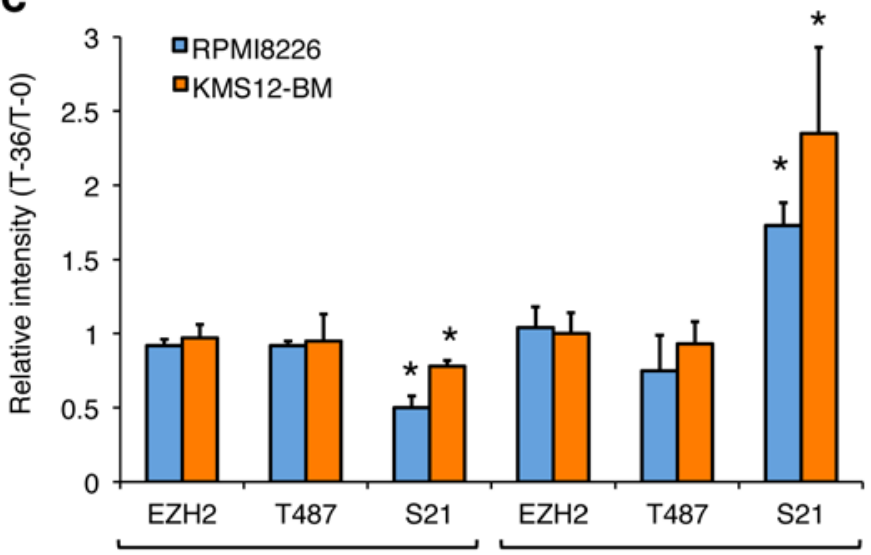

Antroson

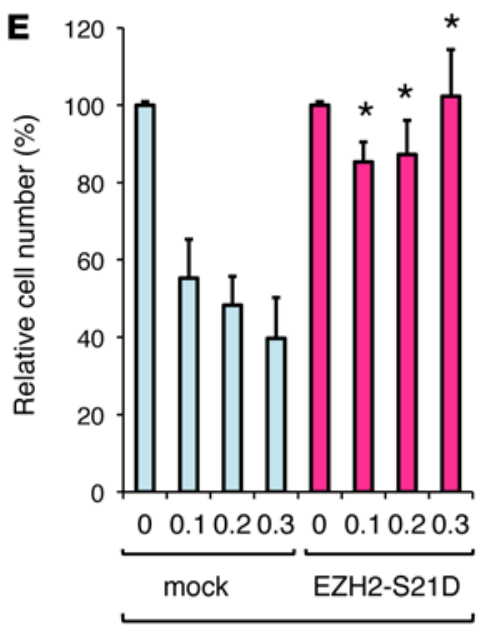

RPMI8226 + ADM $(\mu M)$
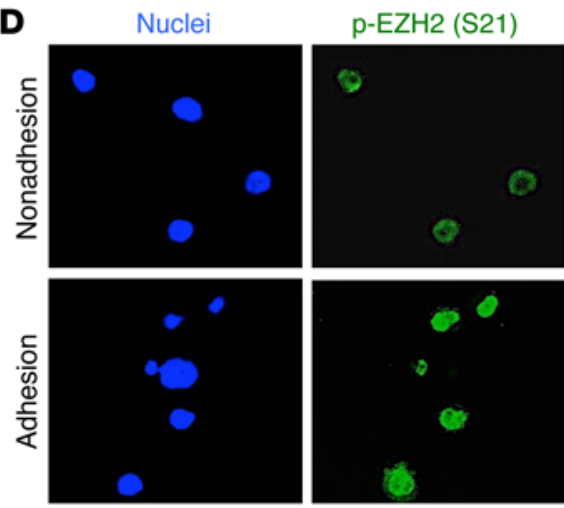

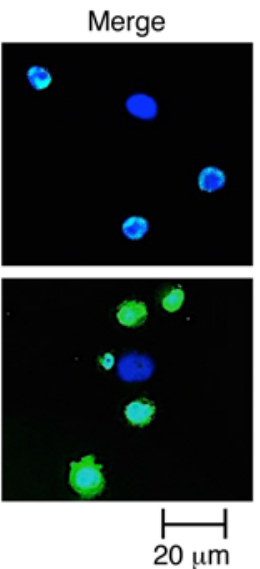

Figure 6. Cell adhesion inactivates EZH2 via S21 phosphorylation, leading to a decrease in $\mathrm{H} 3 \mathrm{~K} 27 \mathrm{me} 3$ in MM cells. (A) Whole cell lysates were prepared after 48 hours of culture described in Figure $2 \mathrm{~A}$ and subjected to immunoblot analysis for EZH2 phosphorylation. (B) RPMI8226 and KMS12-BM cells were cultured in a cell-culture insert with $(+)$ or without (-) direct adhesion to UBE6T-7 cells for 24 hours and a further 36 hours after the addition of $0.4 \mu \mathrm{M}$ ADM. Whole cell lysates were prepared at the indicated time points and subjected to immunoblotting. (C) The signal intensities of each band in panel $\mathbf{B}$ were quantified, normalized to those of the corresponding GAPDH, and shown as relative values with those of $\mathrm{T}-0$ setting at $1.0 .{ }^{*} P<0.05$ against the values of EZH2 determined by 1-way ANOVA with the StudentNewman-Keuls multiple comparisons test $(n=3)$. (D) KMS12-BM cells were cultured in a cell-culture insert with or without direct adhesion to UBE6T-7 cells in the absence of cytotoxic drugs. After 48 hours, cytospin specimens were prepared and stained with an anti-phospho-EZH2 (S21) antibody, followed by staining with Alexa Fluor 488-conjugated anti-rabbit IgG. Nuclei were counterstained with DAPI. Scale bar: $20 \mu \mathrm{m}$. Data shown are representative of 3 independent experiments. (E) RPMI8226 cells were transduced with either the CSII-VENUS (mock) or CSII-VENUS carrying EZH2-S21D (EZH2-S21D) lentiviral vector and cultured with various concentrations of ADM for 72 hours (left panel). ${ }^{*} P<0.05$ against sh-control determined by 1 -way ANOVA with the Student-Newman-Keuls multiple comparisons test $(n=5)$. Whole cell lysates were prepared simultaneously and subjected to immunoblotting (right panel). 
A
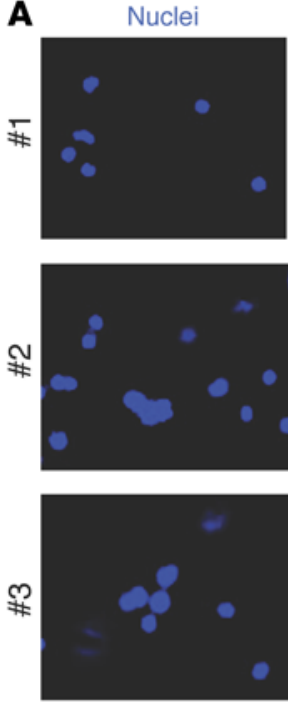

CD138
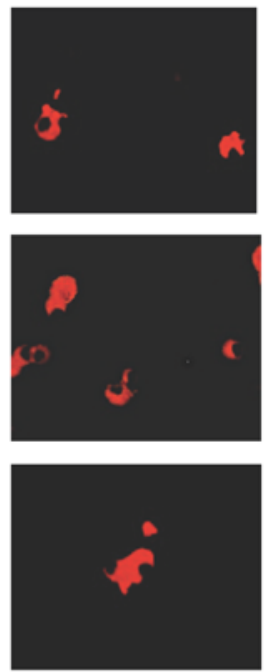

p-EZH2 (S21)
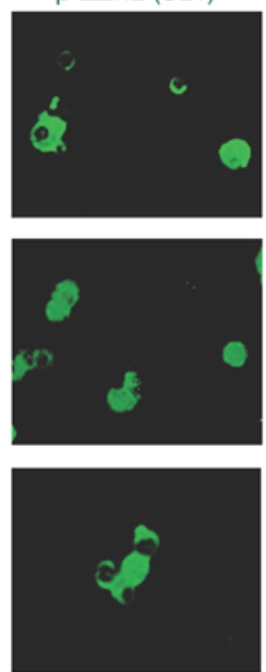

Merge
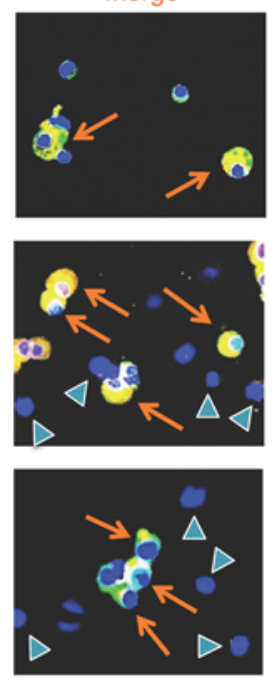

$\underset{20 \mu \mathrm{m}}{\longmapsto}$
B
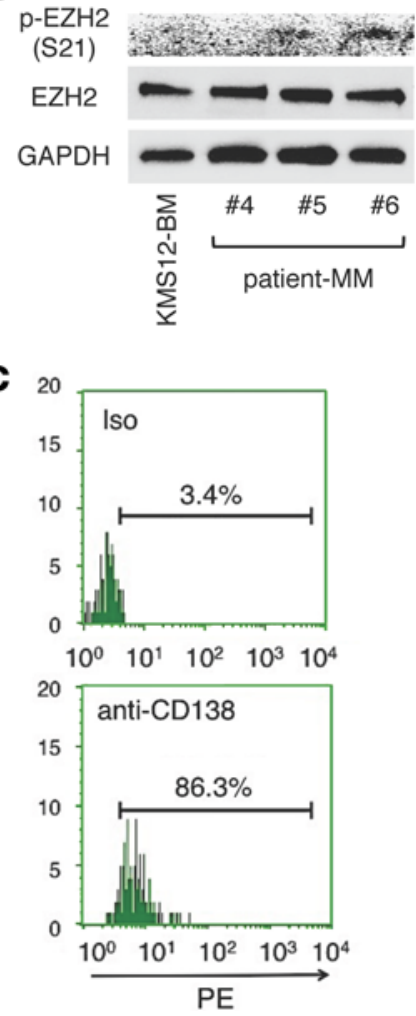

Figure 7. EZH2 was phosphorylated at S21 in CD138-positive BM cells from MM patients. (A) Cytospin specimens of MM patient-derived BM mononuclear cells were incubated with anti-phospho-EZH2 (S21), followed by staining with Alexa Fluor 488-conjugated anti-rabbit IgC and PE-conjugated anti-CD138 antibodies. See Supplemental Figure 5A for antibody validation. Nuclei were counterstained with DAPI. Scale bar: $20 \mu$ m. Data shown are representative of 3 independent experiments (MM patients nos. 1-3). Arrows indicate cells double-positive for CD138 and p-EZH2 (S21); arrowheads indicate cells double-negative for CD138 and p-EZH2 (S21). (B) Whole cell lysates were prepared from CD138-purified BM mononuclear cells from MM patients (no. 4 to no. 6) for immunoblot analysis of EZH2 phosphorylation. KMS12-BM cells cultured under stroma-free conditions serve as a control for underphosphorylated EZH2 at S21. (C) Representative histograms obtained at flow cytometric sorting of CD138-positive cells from BM mononuclear cells of patient no. 6. Over $80 \%$ purity was also yielded in patients no. 4 and no. 5 (data not shown).

EZH2-S21D transduction significantly mitigated ADM-induced cytotoxicity along with the marked reduction of H3K27me3 in the absence of cell adhesion. Among the putative phosphorylation sites of EZH2, we could not evaluate T350 because of the unavailability of specific antibodies against this site. This site is phosphorylated by cyclin-dependent kinase 1 (CDK1) and CDK2, which phosphorylate T492, but do not induce H3K27 hypermethylation $(25,26)$. EZH2-S21 is phosphorylated by Akt kinase (26), which plays a pivotal role in CAM-DR in MM cells (27). A previous study showed that Akt suppresses the methyltransferase activity of EZH2 by impeding its binding to histone H3 (24). Considering these data, we considered S21 as a critical site for regulating EZH2 catalytic activity in CAM-DR induction.

To obtain clinical relevance of the above finding, we examined the phosphorylation status of EZH2 in primary MM cells. $\mathrm{BM}$ mononuclear cells were isolated from $3 \mathrm{MM}$ patients and immediately stained with specific antibodies against CD138 and S21-phosphorylated EZH2 (Figure 7A). EZH2 was phosphorylated at S21 in most CD138-positive MM cells from patients, but was unphosphorylated in CD138-negative non-MM BM cells. EZH2 phosphorylation was maintained in primary MM cells during coculture with BMSCs, but decreased under stroma-free conditions (Supplemental Figure 5C). Immunoblot analyses also revealed EZH2 phosphorylation at S21 in CD138-purified MM cells in 2 out of 3 different cases (Figure 7, B and C). In contrast, EZH2 was not phosphorylated in normal plasma cells purified from healthy volunteers (Supplemental Figure 5). These results indicate that EZH2 phosphorylation at S21 is unique to drugresistant $\mathrm{MM}$ cells in contact with BMSCs and also in primary MM cells localized in the BM. S21-phosphorylated EZH2 appears to be mainly localized in the cytoplasm (Figure 6D and Figure 7A) and thus is unable to access H3K27 in the nuclei. Collectively, the phosphorylation-mediated inactivation of EZH2 appears to promote CAM-DR in MM cells.

Reversal of CAM-DR by kinase inhibitors counteracting EZH2 phosphorylation. Since the catalytic activity of EZH2 was found to be regulated by its phosphorylation, we screened for kinase inhibitors for EZH2 phosphorylation and determined their effects on CAM-DR in MM cells. Using the coculture system, we treated MM cells with suboptimal doses of 21 inhibitors against various kinases (Supplemental Table 2) in the absence or presence of BMSCs. The inhibitors for Akt, CDKs, IGF-1 receptor (IGF-1R), Janus kinase (Jak), and PI3K showed significant cytotoxic activity regardless of direct contact with BMSCs (Figure 8A). We then investigated the 
A

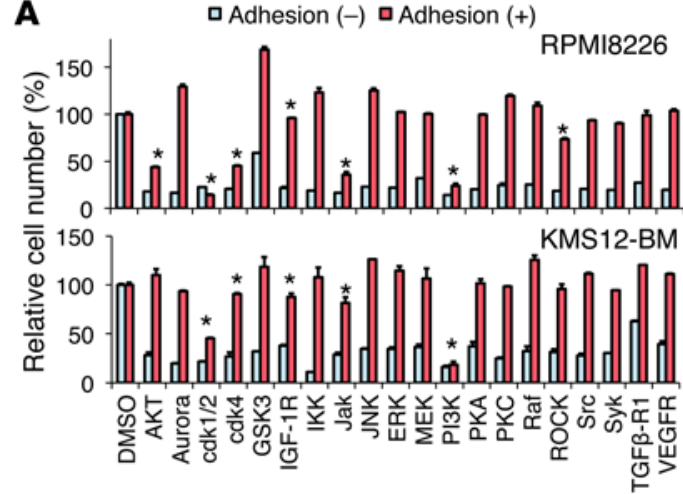

C

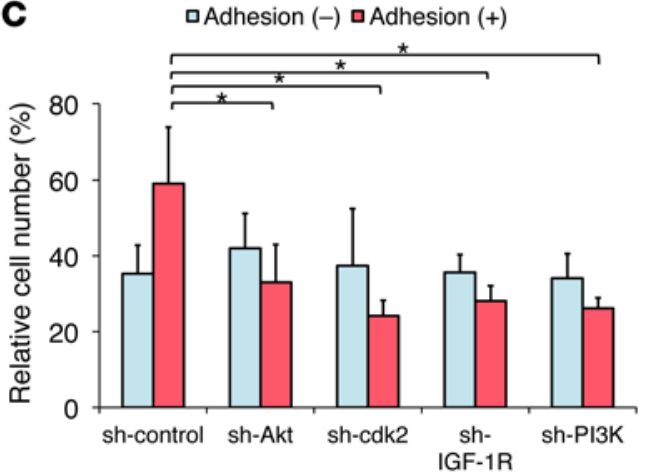

B

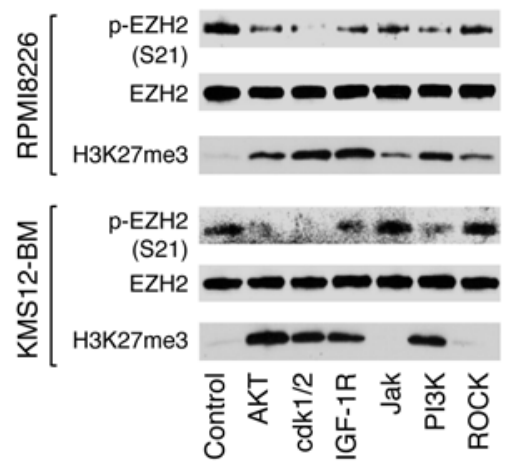

D

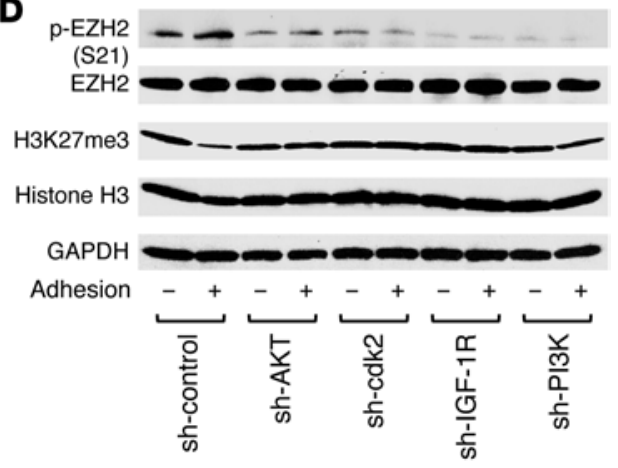

Figure 8. Reversal of CAM-DR by chemical and genetical inhibition of EZH2 phosphorylation. (A) RPMI8226 and KMS12-BM cells were cultured with various kinase inhibitors or vehicle alone (DMSO) in a cell-culture insert with (+) or without (-) adhesion to UBE6T-7 cells for 72 hours. The kinase inhibitors used in this experiment and their doses are listed in Supplemental Table 2. Cell viability was determined by the MTT reduction assay and expressed as a percentage of the values of DMSO-treated cells. ${ }^{*} P<0.05$ against the DMSO control determined by 1 -way ANOVA with the Student-Newman-Keuls multiple comparisons test $(n=3)$. (B) Whole cell lysates were prepared under the adhesion $(+)$ condition of the experiments described in A and subjected to immunoblotting. (C) RPMI8226 cells were transduced with either the pLL3.7-sh-control (sh-control), pLL3.7-sh-Akt\#1 (sh-Akt), pLL3.7-sh-cdk2\#2 (sh-cdk2), pLL3.7-sh-IGF-1R\#2 (sh-IGF-1R), or pLL3.7-sh-PI3K\#1 (sh-PI3K) lentiviral vector, the efficacy of which was validated in pilot experiments (Supplemental Figure 6), and cultured in the absence or presence of ADM for 72 hours. Cell viability was determined by the MTT reduction assay and expressed as a percentage of the values of untreated cells. ${ }^{*} P<0.05$ against sh-control determined by 1-way ANOVA with the Student-Newman-Keuls multiple comparisons test $(n=3)$. (D) Whole cell lysates were prepared after 48 hours of culture described in C and subjected to immunoblotting.

effects of these inhibitors on EZH2 phosphorylation and H3K27 trimethylation. Among these, the inhibitors of Akt, CDK1/2, IGF$1 \mathrm{R}$, and PI3K readily inhibited the phosphorylation of EZH2 and reciprocally upregulated the abundance of $\mathrm{H} 3 \mathrm{~K} 27 \mathrm{me} 3$ in $\mathrm{MM}$ cells (Figure 8B). We confirmed these observations using shRNAmediated knockdown of Akt, CDK2, IGF-1R, and PI3K (see Supplemental Figure 6 for validation) as well as different inhibitors for each target: PD0332991 and AT7519 for CDKs, perifosin and PF-04691502 for PI3K/Akt, and OSI-906 and AG1024 for IGF1R. As anticipated, both shRNA-mediated knockdown and kinase inhibitors effectively overcame CAM-DR along with the cancellation of cell adhesion-mediated EZH2 hyperphosphorylation and H3K27 hypomethylation (Figure 8, C and D, and Figure 9A). Furthermore, the cytotoxic effects of these kinase inhibitors were significantly ameliorated by EZH2 knockdown (Figure 9B), EZH2 inhibitors (Figure 9C), and EZH2-S21D transduction (Figure 9D) along with a decrease in the amounts of H3K27me3 in RPMI8226 cells cocultured with UBE6T-7 cells. These results suggest that the reversal of CAM-DR by kinase inhibitors largely depends on the catalytic activity of EZH2 and subsequent methylation of H3K27. The inhibitors for CDKs and IGF-1R dephosphorylated EZH2 at S21, which was not identified as a direct target of these kinases in previous studies; however, a recent study by Liu et al. (28) demonstrated that CDK2 regulates Akt activity via S477/T479 phosphorylation. In addition, the IGF-1/IGF-1R pathway triggers the activation of PI3K/Akt to enhance the adhesion and migration of MM cells toward stromal cells (29). These observations are supportive of our findings and, when taken together, provide a rationale for listing CDK and IGF-1R inhibitors as candidates of therapeutic agents for overcoming CAM-DR.

Downstream target genes regulated by H3K27 trimethylation during CAM-DR acquisition. Next, we attempted to identify the key molecules regulated by EZH2-mediated H3K27me3 for MM cells to acquire CAM-DR. For this purpose, we diminished the abundance of H3K27me3 in RPMI8226 cells via direct adhesion to BMSCs and evaluated global changes in gene expression using DNA microarrays. As shown in Figure 10A, cell adhesion upregulated several antiapoptotic genes, such as IGF1, chemo- 
A

口Adhesion (-) aAdhesion (+)
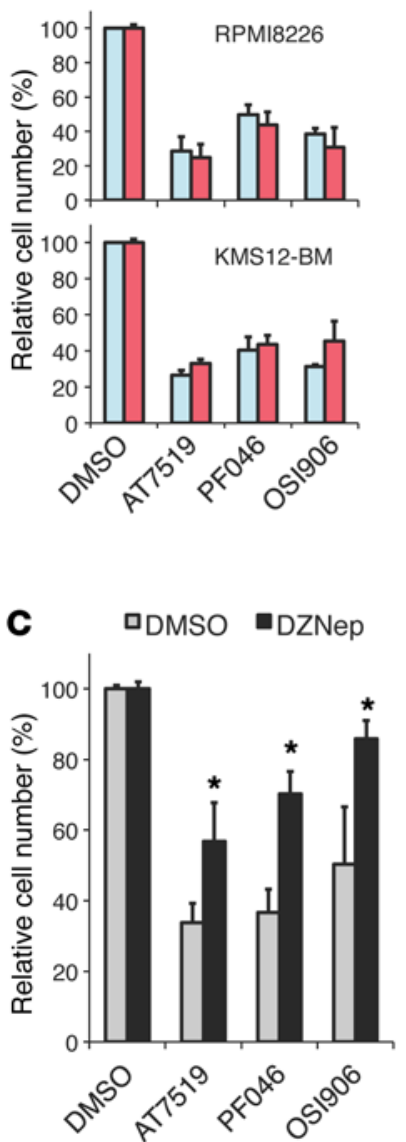

B

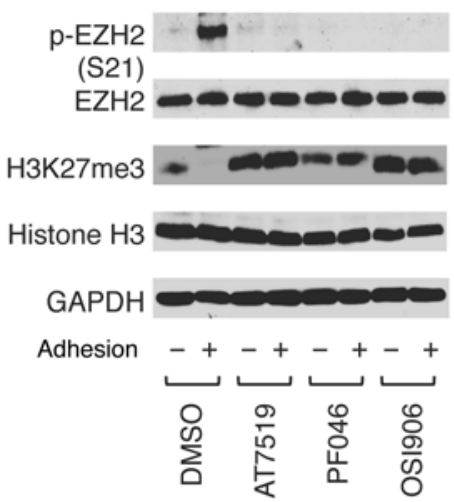

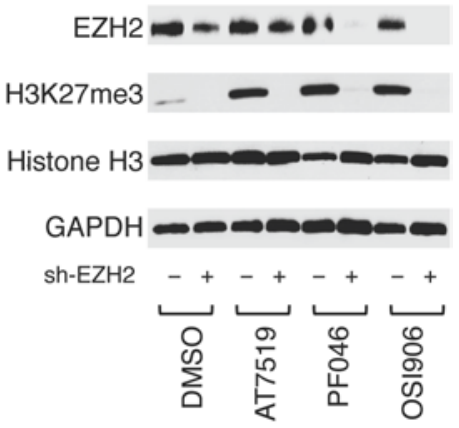
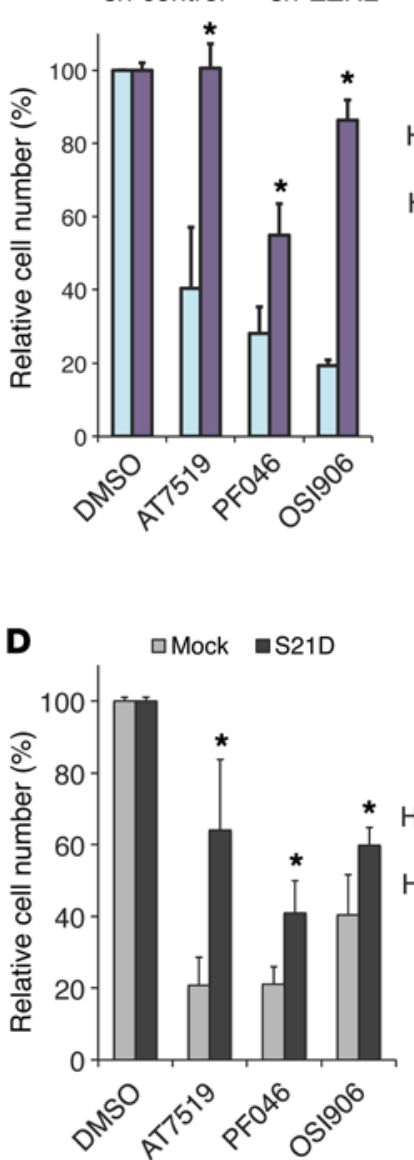

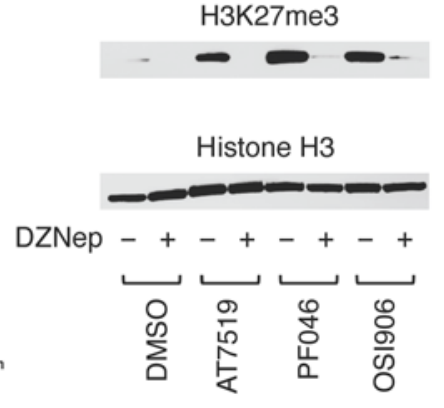

Figure 9. Reversal of CAM-DR by pharmacological and genetic inhibition of EZH2 phosphorylation and its dependence on EZH2 activity. (A) RPMI8226 and KMS12-BM cells were cultured in the presence of the CDK inhibitor AT7519 (0.75 $\mu \mathrm{M})$, the Akt inhibitor PF-04691502 (1.0 $\mu \mathrm{M})$, the IGF-1R inhibitor OSI-906 (1.5 $\mu$ M), or vehicle alone (DMSO) in a cell-culture insert with (+) or without (-) adhesion to UBE6T-7 cells for 72 hours. (B) RPMI8226 cells were transduced with either the pLL3.7-sh-control (sh-control) or the pLL3.7-sh-EZH2 (sh-EZH2) vector and cultured in the presence of AT7519 $(0.75 \mu \mathrm{M})$, PF-04691502 $(1.0 \mu \mathrm{M})$, 0SI-906 $(1.5 \mu \mathrm{M})$, or vehicle alone (DMSO) for 72 hours. (C) RPMI8226 cells were cultured in the presence of AT7519 $(0.75 \mu \mathrm{M})$, PF-04691502 (1.0 $\mu \mathrm{M})$, OSI-906 $(1.5 \mu \mathrm{M})$, or vehicle alone (DMSO) with or without $0.4 \mu \mathrm{M}$ DZNep in a cell-culture insert with adhesion to UBE6T-7 cells for 72 hours. (D) RPMI8226 cells were transduced with either the CSII-VENUS (mock) or CSII-VENUS carrying EZH2-S21D (S21D) lentiviral vector and cultured in the presence of AT7519 (0.75 $\mu \mathrm{M})$, PF-04691502 (1.0 $\mu \mathrm{M})$, $0 \mathrm{SI}-906(1.5 \mu \mathrm{M})$, or vehicle alone (DMSO) for 72 hours. Cell viability was determined by the MTT reduction assay and expressed as a percentage of the values of DMSO-treated cells. Mean \pm SD (bars) of 3 independent experiments are shown in the left panels. ${ }^{*} P<0.05$ against corresponding controls determined by 1 -way ANOVA with the Student-Newman-Keuls multiple comparisons test $(n=3)$. Whole cell lysates were prepared from RPMI8226 cells simultaneously and subjected to immunoblotting (right panels).

kine (C-X-C motif) ligand 2 (CXCL2), B cell CLL/lymphoma 2 (BCL2), and hypoxia inducible factor 1, a subunit (HIF1A), whereas no significant differences were observed for $M Y C$, HDAC1, SP1, KLF9, NEK2, IRF4, HOXA9, CDK2, and ITGA4, all of which are known to be antiapoptotic in MM cells $(11,30)$. Next, we carried out a reciprocal experiment in which H3K27 hypermethylation was induced by a treatment with the PI3K/Akt inhibitor PF-04691502. In microarray analyses, PF-04691502 reciprocally downregulated the expression of IGF1, CXCL2, $B C L 2$, and HIF1A as well as several other antiapoptotic genes, such as IRF4, HOXA9, CDK2, ITGA4, ITGB7, and IL6R. The results of microarray analyses were confirmed by semiquantitative reverse-transcription PCR (RT-PCR); direct adhesion increased the expression levels of IGF1, BCL2, and HIF1A, but did not affect those of IRF4 and MYC in RPMI8226 and KMS12-BM cells (Figure 10B). We then performed ChIP assays to determine the mechanisms by which cell adhesion regulates the transcription of these genes. We examined the methylation status of $\mathrm{H} 3 \mathrm{~K} 27$ in the promoter regions of the IGF1, BCL2, and IRF 4 genes according to previous studies (31-33). As anticipated, H3K27 was heavily methylated in the promoter regions of IGF1 and BCL2 in ADM-treated RPMI8226 cells without cell adhesion, whereas it was hypomethylated under contact with BMSCs even after the treatment with ADM (Figure 11A). In contrast, cell adhesion did not affect the methylation status in the promoter region of the IRF 4 gene. These data directly link the methylation status of H3K27 to cell adhesion-regulated antiapoptotic genes such as IGF1 and BCL2. 
A

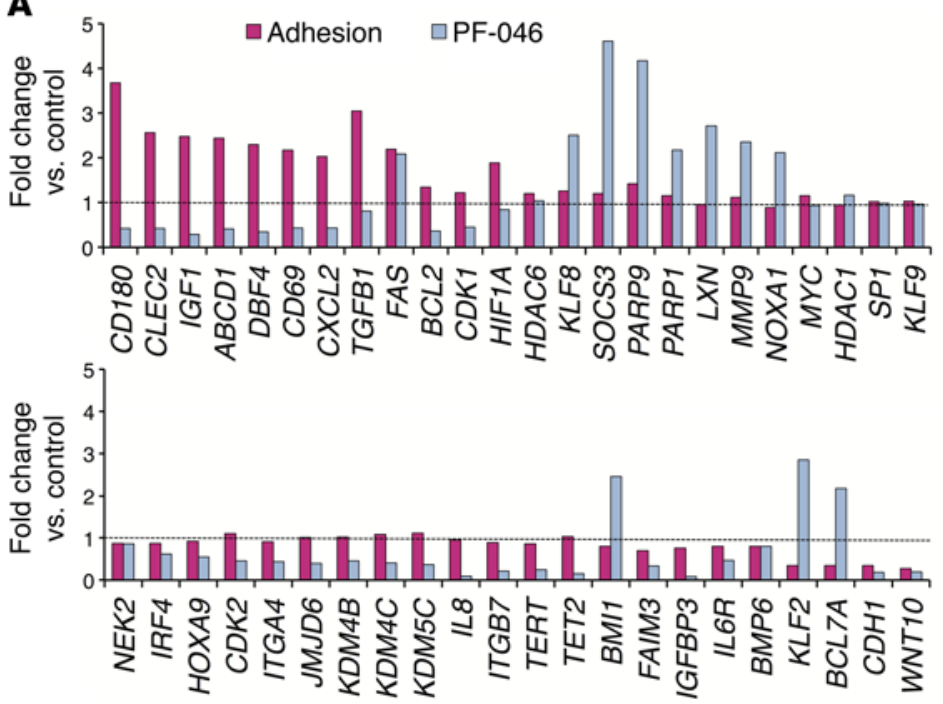

B

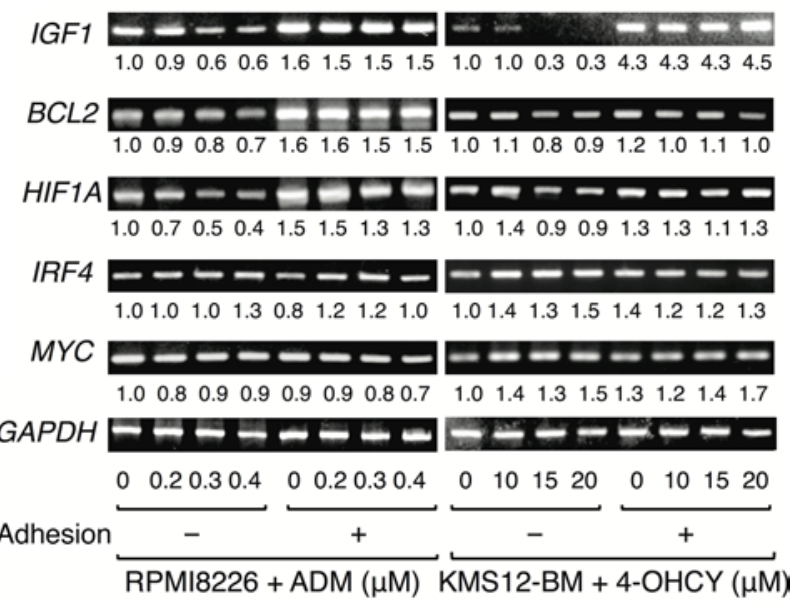

Figure 10. Downstream targets regulated by the modulation of H3K27me3 during CAM-DR induction. (A) RPMI8226 cells were cultured with or without adhesion to UBE6T-7 cells or in the absence or presence of $1.0 \mu \mathrm{M}$ PF-04691502 for 24 hours for microarray analyses. The $y$ axis shows the relative expression levels of the indicated genes with those of RPMI8226 cells cultured without adhesion as a reference. We considered the changes significant when the expression was increased or deceased more than 2-fold with an FDR threshold of 0.05. (B) Total cellular RNA was isolated from RPMI8226 and KMS12-BM cells in the experiments described in Figure 2A and subjected to semi-quantitative RT-PCR analysis for the indicated genes. See Supplemental Table 1 for primer sequences. The results of suboptimal amplification cycles (35 cycles) are shown.

A recent report by Chiron et al. (34) implicating IGF-1 as an autocrine activator of MM urged us to investigate the role of IGF-1 for CAM-DR using gain- and loss-of-function approaches. We used recombinant IGF-1 (rIGF-1) to examine its effects on druginduced cytotoxicity under stroma-free conditions. Cell proliferation assays revealed that rIGF-1 almost completely cancelled 4-OHCY-induced cytotoxicity in RPMI8226 cells along with the marked upregulation of EZH2 phosphorylation at S21 and concomitant disappearance of the H3K27me3 mark (Figure 11B). In a reciprocal experiment, IGF1 knockdown significantly enhanced ADM-induced cytotoxicity along with a marked reduction in EZH2 phosphorylation and reciprocal hypermethylation of H3K27 in the presence of BMSCs (Figure 11C and Supplemental Figure 7A). Indeed, IGF-1 secretion by MM cells was significantly increased via direct contact with BMSCs (Supplemental Figure 7B). These results suggest that IGF-1 is a critical downstream target of H3K27 hypomethylation during the induction of CAM-DR.

In the present study, we identify what we believe to be a novel epigenetic mechanism for CAM-DR in MM cells. Our results, together with previous findings, illustrate a mechanistic view of CAM-DR from the membrane to nucleus signaling, including the regulatory circuit of IGF-1 (Figure 11D). In response to adhesion signals transduced from the surface adhesion molecule VLA-4, the PI3K/Akt pathway is initially activated to induce EZH2 phosphorylation in MM cells. The phosphorylation-mediated inactivation of EZH2 results in the expression of antiapoptotic genes, such as IGF1, BCL2, and HIF1A, via H3K27 hypomethylation at their promoter regions. Secreted IGF-1 additively activates the IGF-1/IGF-1R pathway in an autocrine manner, further enhancing the activation of the PI3K/Akt pathway. Consistent with this view, we demonstrate that direct adhesion initially induced
Akt-S473 phosphorylation, followed by IGF-1R phosphorylation. In addition, CDK1/2 and IGF-1R inhibitors downregulated Akt-S473 phosphorylation; this was followed by EZH2 dephosphorylation (data not shown).

Therapeutic effects of the IGF-1R inhibitor OSI-906 in murine models of refractory $M M$. The above findings prompted us to investigate the possibility of kinase inhibitors, such as PI3K/ Akt, IGF-1R, and CDK inhibitors, counteracting EZH2 phosphorylation to overcome CAM-DR of MM. Based on our proposal of the critical role of the IGF-1/IGF-1R pathway, we examined the efficacy of the specific IGF-1R inhibitor OSI-906 in subsequent experiments. First, we determined the combined effects of OSI-906 with conventional anti-MM drugs in the presence of BMSCs in vitro. An isobologram analysis of drug interactions revealed that OSI-906 exerted additive to synergistic cytotoxicity with 4-OHCY, ADM, and L-PAM (Figure 12A), with the enhancement of drug-induced EZH2 dephosphorylation and H3K27 hypermethylation, both of which were supposed to be inhibited by cell adhesion (Figure 12B). The latter finding strongly suggests the potential of OSI-906 to overcome CAM-DR. To test this possibility in vivo, we established 2 murine models of refractory MM recapitulating CAM-DR. The first model used immunodeficient mice injected subcutaneously with human MM cells in either the absence or presence of BMSCs. Specifically, RPMI8226-Luc and UBE6T-7 cells were injected separately in the right and left thighs, respectively (separate inoculation), or injected as a mixture in the right thigh (mixed injection) of NOD/SCID mice (Figure 13A). The administration of cyclophosphamide (CY) alone, OSI-906 alone, and their combination significantly reduced the viability of MM cells, as determined by luciferase activity, in the separate inoc- 
A

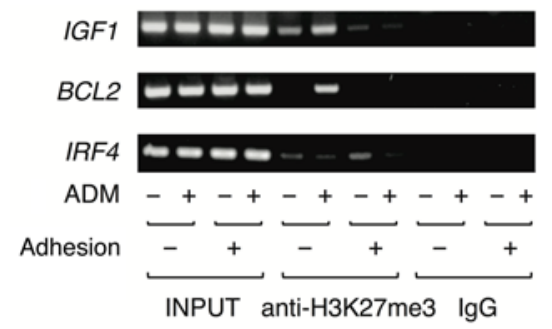

B

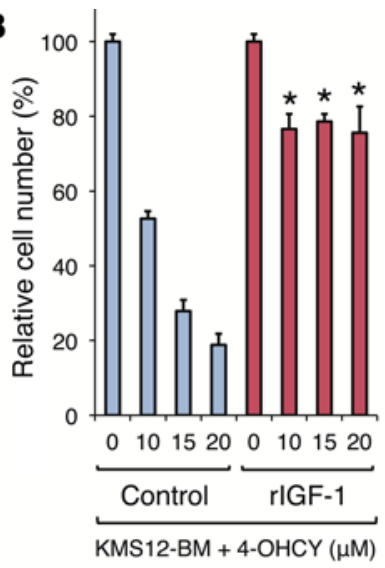

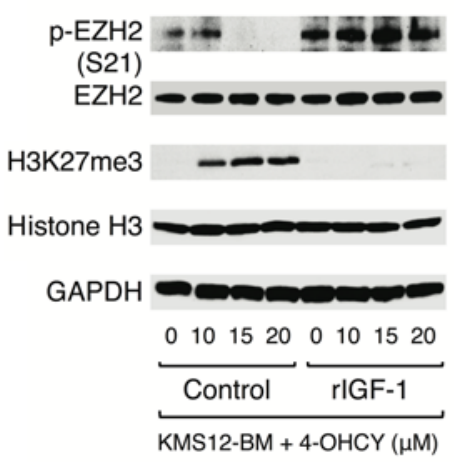
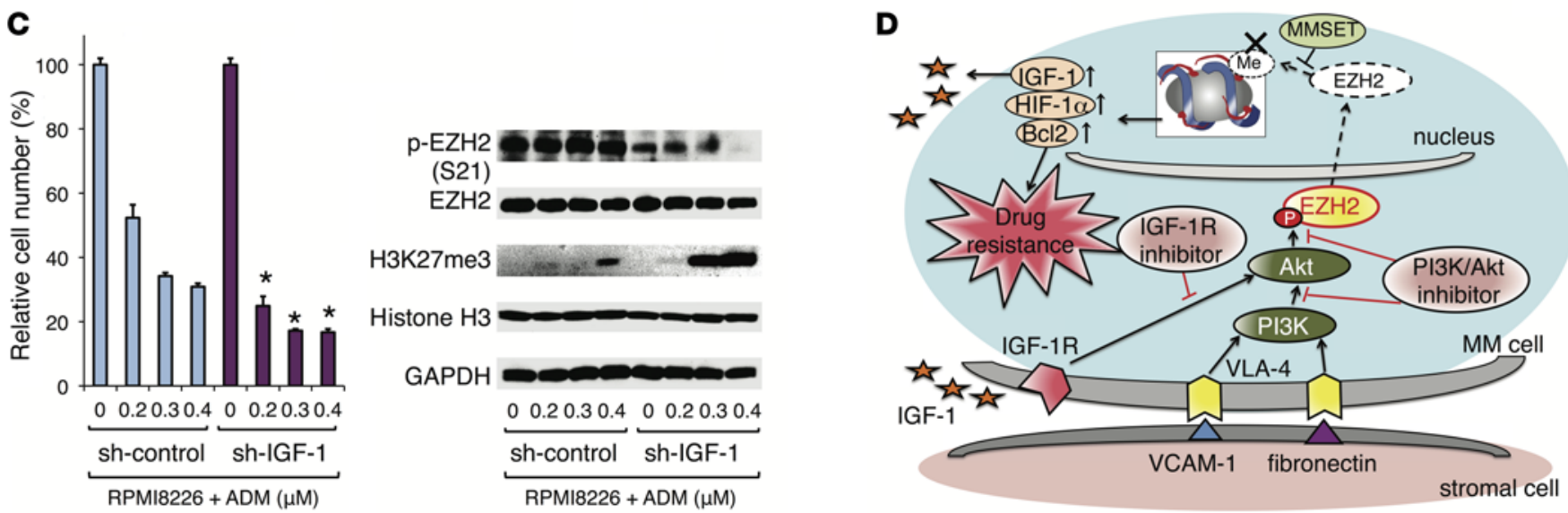

Figure 11. IGF-1 is a downstream target of H3K27 hypomethylation and a key mediator of CAM-DR. (A) Chromatin suspensions were prepared from RPMI8226 cells in the experiments described in Figure 2A and immunoprecipitated with anti-H3K27me3 and isotype-matched (IgG) antibodies. The resulting precipitants were subjected to PCR to amplify the promoter regions of the IGF1, BCL2, and IRF4 genes (31-33), using primers listed in Supplemental Table 4. Representative data of 50 cycles are shown. Input indicates that PCR was performed with genomic DNA. (B) RPMI8226 cells were cultured with rIGF-1 at $100 \mathrm{ng} / \mathrm{ml}$ or vehicle (control) in the absence or presence of 4-OHCY under stroma-free conditions. Cell viability was determined by the MTT reduction assay after 72 hours (left panel). ${ }^{*} P<0.05$ against controls at the same concentrations of 4-OHCY $(n=3)$. Whole cell lysates were prepared simultaneously and subjected to immunoblotting (right panel). (C) RPMI8226 cells were transduced with either the pLL3.7-sh-control (sh-control) or pLL3.7-sh-IGF-1 (sh-IGF-1) vector, whose efficacy was validated by RT-PCR (Supplemental Figure 7), and cultured in the absence or presence of ADM for 72 hours. Cell proliferation was measured by the MTT reduction assay (left panel). ${ }^{*} P<0.05$ against sh-controls at the same concentrations of ADM $(n=3)$. Whole cell lysates were prepared simultaneously and subjected to immunoblotting (right panel). (D) The role of the IGF-1R/PI3K/Akt pathway in epigenetic regulation of CAM-DR in MM cells. See Results and Discussion for details.

ulations (Figure 13, A and B). In striking contrast, CY alone was not cytotoxic to MM cells injected together with stromal cells, indicating that the refractoriness to $\mathrm{CY}$ was due to CAM-DR (Figure 13, A and B). Even under the "mixed" condition, however, OSI-906 significantly reduced the tumor burden as a single agent and, more importantly, had an additive effect with CY. Next, we confirmed the ability of OSI-906 to overcome CAMDR using another murine model, in which MOPC315.BM.Luc murine MM cells were injected intravenously into syngeneic BALB/c mice. Because MOPC315.BM.Luc cells engraft in the $\mathrm{BM}$ and interact with the $\mathrm{BM}$ microenvironment, this model accurately reflects human disease and is considered suitable for tropism analysis and drug testing (35-37). Using this model, we confirmed that CY was not able to inhibit the growth of MM cells engrafted in the BM, whereas OSI-906 alone significantly reduced tumor growth (Figure 14, A and B). More importantly,
OSI-906 clearly exhibited synergy with an ineffective dose of CY. The therapeutic effect of OSI-906 was validated in histopathological examination, in which BM was hypercellular with the infiltration of MOPC315.BM.Luc cells in control (data not shown) and CY-treated mice (Figure 14C). In contrast, cellularity was moderately reduced by OSI-906 treatment (Figure 14C). Immunofluorescent staining revealed that EZH2 was strongly phosphorylated in CD138-positive MM cells refractory to CY, whereas phosphorylation was almost completely abrogated in those treated with OSI906 in vivo (Figure 14C). In an unengrafted area of CY-treated mice, neither EZH2 phosphorylation nor CD138 positivity was detected, indicating the reliability and specificity of the method (Figure 14C). Finally, we confirmed whether EZH2 dephosphorylation at S21 mediates the ability of OSI-906 in overcoming drug resistance in vivo using EZH2-S21D-transduced MOPC315. BM.Luc cells in the same system. As anticipated, EZH2-S21D 
A

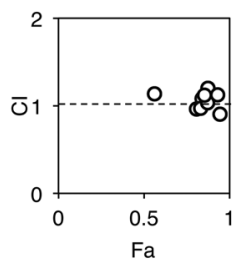

4-OHCY

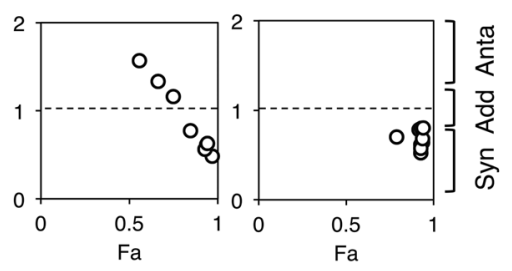

ADM

L-PAM

B

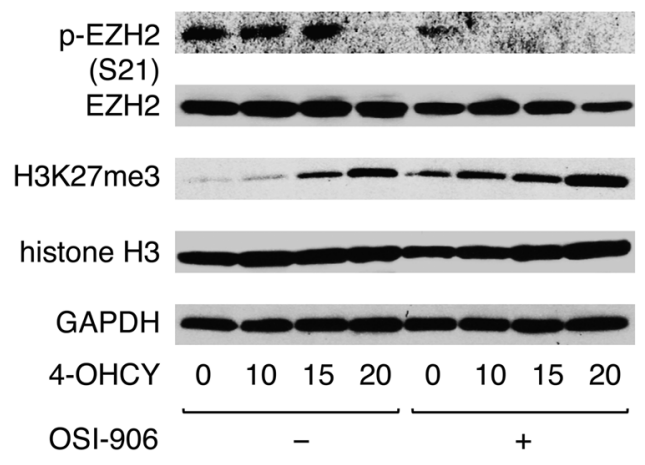

transduction almost completely abrogated the antitumor effect of OSI-906 (Supplemental Figure 8). These results suggest that OSI-906 exerted cytotoxicity against cell adhesion-mediated drug-resistant MM cells via the dephosphorylation of EZH2 in vivo. Kinase inhibitors counteracting EZH2 phosphorylation may thus be effective for overcoming CAM-DR in MM patients and improving their treatment outcome.

\section{Discussion}

In the present study, we have demonstrated that phosphorylation-mediated EZH2 inactivation and subsequent decreases in H3K27me3 levels are related to CAM-DR in MM cells. This is the first report, to our knowledge, of the epigenetic mechanism for CAM-DR involving a regulatory circuit from the membrane to the nucleus (Figure 11D). In accordance with this model, small compounds counteracting EZH2 phosphorylation, such as PI3K/ Akt, CDK, and IGF-1R inhibitors, were found to be effective for the reversal of CAM-DR in vitro. Of these, the IGF-1R inhibitor OSI-906 was able to overcome CAM-DR in mouse models, which may be translated to the clinic to improve the treatment outcome of MM patients in combination with conventional antiMM agents. In support of this view, the potent and selective cytotoxicity of IGR-1R inhibitors against MM cells was clearly demonstrated by a recent systematic pharmacological screen using 119 cell lines from more than 10 different types of hematologic malignancies (38). As anticipated, PI3K and Akt inhibitors were also effective for MM cells, albeit with lower selectivity than IGF-1R inhibitors (Supplemental Figure 9).

The present findings may have broader significance because EZH 2 is frequently overexpressed and dysregulated in multiple types of cancer. The expression level of EZH2 is correlated with the higher proliferation rates and aggressive behavior of cancer cells as well as the poor prognosis of patients with breast, prostate, and bladder cancers (22). In contrast, EZH2 functions as a tumor suppressor in some types of cancer. Inacti-
Figure 12. The IGF-1R inhibitor OSI-906 overcomes CAM-DR in vitro. (A) An isobologram analysis of the interaction between OSI-906 and conventional anti-MM drugs (4-OHCY, ADM, and L-PAM) using RPMI8226 cells in the presence of UBE6T-7 cells. See the legend of Figure 4, D and E, for the isobologram method. (B) Immunoblot analysis was performed simultaneously with the experiments shown in $\mathbf{A}$. Representative results of the combination of OSI-906 and 4-OHCY. vating mutations of $E Z H 2$ were found in patients with myeloid malignancies, including myelodysplastic syndrome and myeloproliferative neoplasms, and have been associated with poor patient survival $(39,40)$. In line with this observation, mice with conditional deletions of EZH2 and TET2 in hematopoietic stem cells develop myelodysplastic syndrome and myeloproliferative neoplasms $(41,42)$. Loss-of-function mutations and deletions of the EZH2 and SUZ12 genes have been reported in $25 \%$ of $\mathrm{T}$ cell-acute lymphoblastic leukemias (43). Consistent with this finding, the conditional deletion of EZH2 in BM cells causes $\mathrm{T}$ cell leukemia (44). These results suggest that the oncogenic role of EZH2 is cell-context dependent and differs between solid tumors and hematological malignancies. EZH2 is not mutated or deleted in MM; therefore, it can integrate environmental cues to biological output-enhancing malignant phenotypes in this disease. From a mechanistic standpoint, it is interesting that EZH2 methylates cellular proteins other than histone $\mathrm{H} 3$ and modulates their functions. For example, Kim et al. (45) showed that EZH2 methylated STAT3 and upregulated its transcriptional activity, contributing to the maintenance of stem cell phenotypes and tumor progression of glioblastoma. Our data show that EZH2 is central to tumor biology, from the initiation of cancer to drug resistance, and thus is a major therapeutic target in various cancers.

\section{Methods}

In vitro coculture system with BMSCs to recreate the BM microenvironment. We used a culture system devised from a modified cell-culture insert of Kawada et al. (46). First, BMSCs were cultured on the reverse side of the polyethylene terephthalate track-etched membrane of a cell-culture insert (35-3495, high pore density cell-culture insert; BD) in a 24-well plate (35-3504; BD). After obtaining a confluent feeder layer, MM cells were seeded on the upper side of the membrane of the insert where the cytoplasmic villi of BMSCs passed through the etched $0.4-\mu \mathrm{m}$ pores. In another set of conditions, BMSCs were cultured at 
A
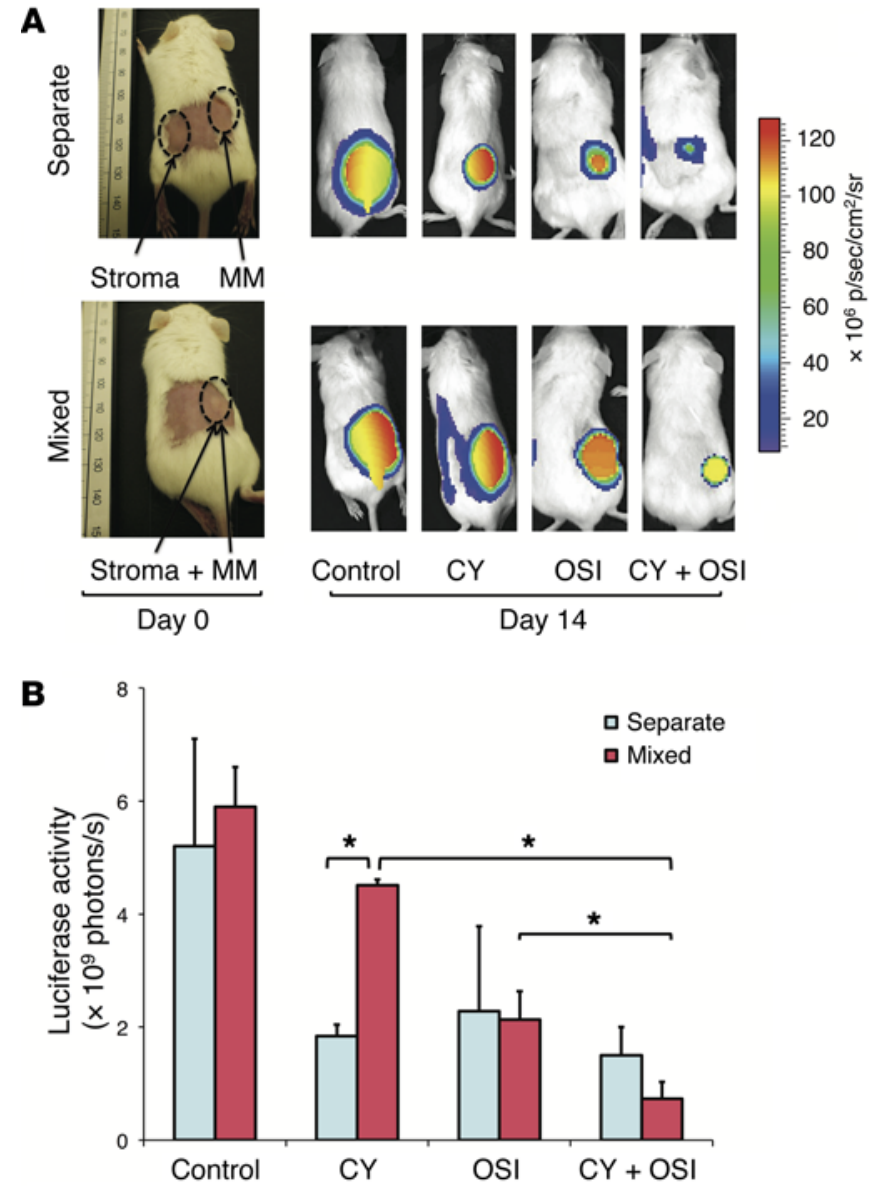

the bottom of wells in a 24-well plate (35-3504; BD). After obtaining a confluent feeder layer, MM cells were seeded on the upper side of the insert (35-3095, low pore density cell-culture insert; BD). Under these conditions, MM cells were physically separated from the stromal layer, providing an adhesion-negative control (14).

Xenogeneic and syngeneic murine MM models. For ex vivo tracing of tumors, we established luciferase-expressing sublines of RPMI8226 and MOPC315.BM, designated RPMI8226-Luc and MOPC315.BM.Luc, respectively, by transfecting firefly luciferase cDNA (35). Cells were suspended in $100 \mu \mathrm{l}$ of RPMI 1640 medium before injection. RPMI8226-Luc cells were inoculated subcutaneously in the right and left thighs of NOD/SCID mice (Charles River Laboratories) (47). MOPC315.BM.Luc cells were injected intravenously into BALB/c mice (Charles River Laboratories) (35). Drugs were administered intraperitoneally in a $200 \mu \mathrm{l}$ volume of solution containing 5\% DMSO and $95 \%$ sterile $0.9 \% \mathrm{NaCl}$ twice a week at $30 \mathrm{mg} / \mathrm{kg}$ for 2 weeks as described previously (48). The control group received the vehicle $(5 \% \mathrm{DMSO}$ in $0.9 \% \mathrm{NaCl}$ ) alone on the same schedule. Tumor burden was monitored by measuring tumorderived luciferase activity with the noninvasive bioimaging system. In short, tumor-bearing mice were injected with $1.5 \mathrm{mg}$ of the luciferase substrate D-luciferin intraperitoneally after being anesthetized with isoflurane. Photons transmitted through the body were collected for a specified length of time and analyzed using the IVIS Imaging System with Living Image software (Xenogen). Quantitative data were expressed as photon units (photons/s) (49).
Figure 13. The IGF-1R inhibitor 0SI-906 overcomes CAM-DR in a xenograft murine MM model. (A) We subcutaneously inoculated $5 \times 10^{6}$ luciferaseexpressing RPMI8226-Luc cells and $5 \times 10^{6}$ UBE6T-7 cells separately in the right and left thighs, respectively (separate) or a mixture of the 2 cell types in the right thigh (mixed) of NOD/SCID mice. Mice were treated in 4 ways: vehicle alone (control), $30 \mathrm{mg} / \mathrm{kg} \mathrm{CY}, 30 \mathrm{mg} / \mathrm{kg} 0 \mathrm{OI}-906$ (OSI), and a combination of both agents $(\mathrm{CY}+\mathrm{OSI})$. Treatments were started when inoculated tumors were measurable, defined as day 0 . On day 14 , in vivo luciferase activity of whole body was measured by o-luciferin injection and the IVIS Imaging System. Representative photographs of NOD/SCID mice on day 0 and day 14 are shown. Original magnification, $\times 2$. Inoculated tumors on day 0 are indicated in circled regions. (B) Quantitative data of in vivo bioluminescence imaging on day 14 expressed as photon units (photons/s). ${ }^{*} P<0.05$ determined by 1 -way ANOVA with the Tukey's multiple comparison test $(n=3)$.

Microarray data. All original microarray data were deposited in the NCBI's Gene Expression Omnibus (GEO GSE66466).

Statistics. We used 1-way ANOVA with the Student-NewmanKeuls multiple comparisons test to determine statistical significance. $P$ values of less than 0.05 were considered significant.

Study approval. Before the isolation of samples from patients and healthy volunteers, informed consent was obtained in accordance with the Declaration of Helsinki and the protocol was approved by the Institutional Review Board of Jichi Medical University. All animal studies were approved by the Institutional Animal Ethics Committee and performed in accordance with the Guide for the Care and Use of Laboratory Animals formulated by the National Academy of Sciences.

\section{Author contributions}

JK designed and performed experiments, analyzed data, and drafted the manuscript; DK and TW performed experiments; TI provided clinical samples; $\mathrm{POH}$ and BB provided MOPC315.BM.Luc cells and critically reviewed the manuscript; and YF designed and supervised research and wrote the manuscript.

\section{Acknowledgments}

The authors are grateful to Kiyoshi Ando (Tokai University, Tokyo, Japan) for his technical advice regarding the establishment of the coculture system and Akiko Yonekura for her technical assistance. This work was supported in part by the High-Tech 

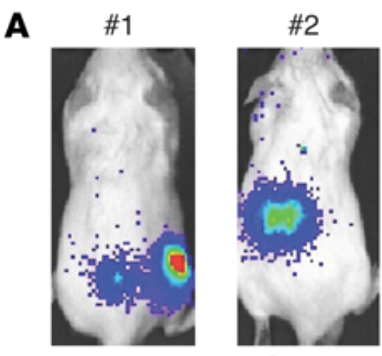

Control

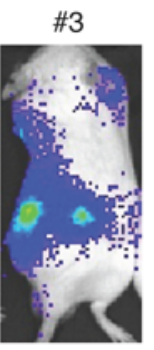

\#3

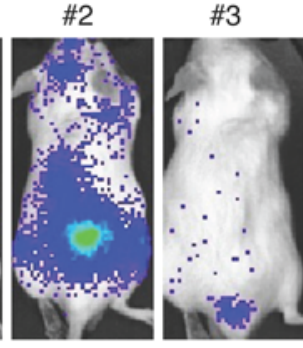

OSI

$\mathbf{B}$

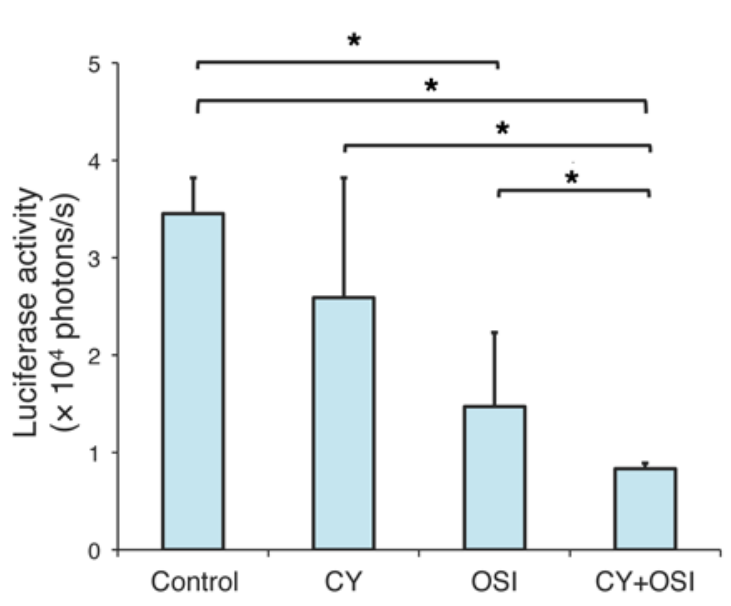

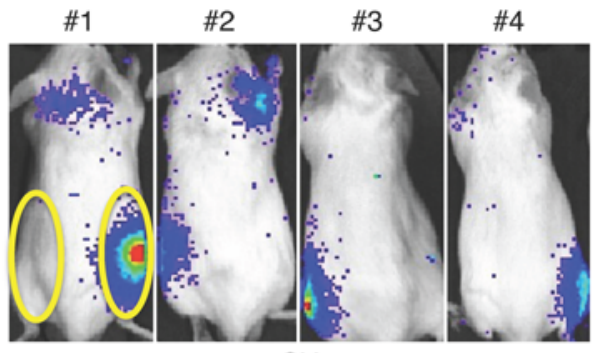

CY
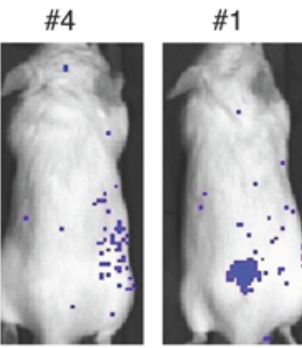

\#2

\#3

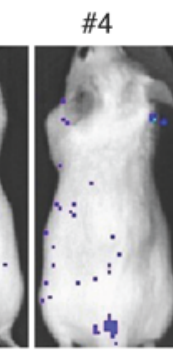

$\mathrm{CY}+\mathrm{OSI}$

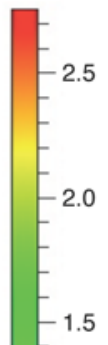

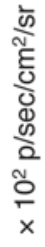

C
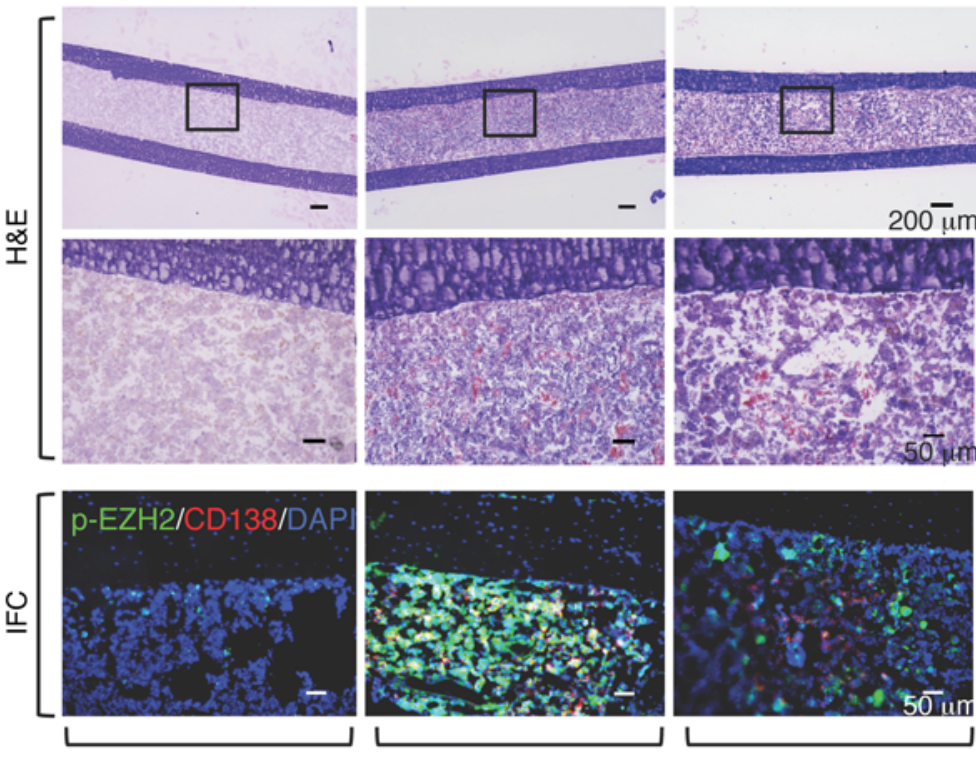

CY\#1-left

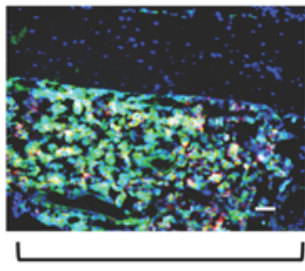

CY\#1-right

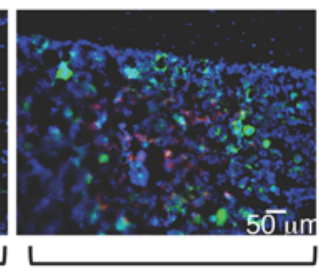

OSI\#1

Figure 14. The IGF-1R inhibitor OSI-906 overcomes CAM-DR in a syngeneic murine MM model. (A) We injected $2 \times 10^{5}$ luciferase-expressing MOPC315. BM. Luc cells intravenously into BALB/c mice and treated them in 4 ways: the vehicle alone (control), $30 \mathrm{mg} / \mathrm{kg} \mathrm{CY,} 30 \mathrm{mg} / \mathrm{kg} 0 \mathrm{SI}-906$ (OSI), and the combination of both agents $(C Y+O S I)$. Treatments were started 2 weeks after transplantation, defined as day 0 . In vivo luciferase activity of whole body was measured by the IVIS Imaging System on day 14. Photographs of all BALB/c mice on day 14 are shown. Original magnification, $\times 2$. (B) Quantitative data of in vivo bioluminescence imaging expressed as photon units. ${ }^{*} P<0.05$ determined by 1-way ANOVA with the Tukey's multiple comparison test ( $n=3$ ). (C) BM sections were obtained from the femurs corresponding to the circled regions in A and subjected to H\&E and immunofluorescent chemical (IFC) staining. Immunofluorescent chemical specimens were stained with anti-phospho-EZH2 at S21 (p-EZH2), followed by staining with Alexa Fluor 488-conjugated anti-rabbit IgC (green), and PE-conjugated anti-mouse CD138 (red) antibodies. Nuclei were counterstained with DAPI (blue). Only merged images are shown. Scale bars: $200 \mu \mathrm{m}$ (upper panels); $50 \mu \mathrm{m}$ (middle and lower panels). Data shown are representative results of 3 independent experiments.

Research Center Project for Private Universities: a Matching Fund Subsidy from MEXT, Grant-in-Aid for Scientific Research from the Japan Society for the Promotion of Science (grants 25118721, 25461434, and 26461438), and research grants from the Japan Leukemia Research Fund, the Yasuda Memorial Cancer Foundation, and the Takeda Science Foundation (to J. Kikuchi and Y. Furukawa). J. Kikuchi and Y. Furukawa received a Kano
Foundation Research grant and the Award in Aki's Memory, respectively, from the International Myeloma Foundation Japan.

Address correspondence to: Yusuke Furukawa, Division of Stem Cell Regulation, Center for Molecular Medicine, Jichi Medical University, 3311-1 Yakushiji, Shimotsuke, Tochigi 329-0498, Japan. Phone: 81.285.58.7399; E-mail: furuyu@jichi.ac.jp. 
1. Nefedova Y, Landowski TH, Dalton WS. Bone marrow stromal-derived soluble factors and direct cell contact contribute to de novo drug resistance of myeloma cells by distinct mechanisms. Leukemia. 2003;17(6):1175-1182.

2. Hideshima T, Anderson KC. Novel therapies in MM:from the aspect of preclinical studies. Int $J$ Hematol. 2011;94(4):344-354.

3. Black JC, Van Rechem C, Whetstine JR. Histone lysine methylation dynamics:establishment, regulation, and biological impact. Mol Cell. 2012;48(4):491-507.

4. Greer EL, Shi Y. Histone methylation:a dynamic mark in health, disease and inheritance. Nat Rev Genet. 2012;13(5):343-357.

5. Sharma SV, et al. A chromatin-mediated reversible drug-tolerant state in cancer cell subpopulations. Cell. 2010;141(1):69-80.

6. Roesch A, et al. Overcoming intrinsic multidrug resistance in melanoma by blocking the mitochondrial respiration chain of slow-cycling JARID1B ${ }^{\text {high }}$ cells. Cancer Cell. 2013;23(6):811-825.

7. Zheng Y, et al. Total kinetic analysis reveals how combinatorial methylation patterns are established on lysines 27 and 36 of histone H3. Proc Natl Acad Sci U S A. 2012;109(34):13549-13554.

8. Popovic R, et al. Histone methyltransferase MMSET/NSD2 alters EZH2 binding and reprograms the myeloma epigenome through global and focal changes in H3K36 and H3K27 methylation. PLoS Genet. 2014;10(9):e1004566.

9. Lauring J, et al. The multiple myeloma-associated MMSET gene contributes to cellular adhesion, clonogenic growth, and tumorigenicity. Blood. 2008;111(2):856-864.

10. Brito JL, et al. MMSET deregulation affects cell cycle progression and adhesion regulons in t(4;14) myeloma plasma cells. Haematologica. 2009;94(1):78-86

11. Martinez-Garcia E, et al. The MMSET histone methyl transferase switches global histone methylation and alters gene expression in $\mathrm{t}(4 ; 14) \mathrm{mul}-$ tiple myeloma cells. Blood. 2011;117(1):211-220.

12. Schwartzentruber J, et al. Driver mutations in histone $\mathrm{H} 3.3$ and chromatin remodelling genes in paediatric glioblastoma. Nature. 2012;482(7384):226-231.

13. Bender S, et al. Reduced H3K27me3 and DNA hypomethylation are major drivers of gene expression in K27M mutant pediatric high-grade gliomas. Cancer Cell. 2013;24(5):660-672.

14. Kikuchi J, Koyama D, Mukai HY, Furukawa Y. Suitable drug combination with bortezomib for multiple myeloma under stroma-free conditions and in contact with fibronectin or bone marrow stromal cells. Int J Hematol. 2014;99(6):726-736.

15. De Schauwer C, Meyer E, Van de Walle GR, Van Soom A. Markers of stemness in equine mesenchymal stem cells: a plea for uniformity. Theriogenology. 2011;75(8):1431-1443.

16. Cheng MF, Lee CH, Hsia KT, Huang GS, Lee HS. Methylation of histone H3 lysine 27 associated with apoptosis in osteosarcoma cells induced by staurosporine. Histol Histopathol. 2009;24(9):1105-1111.

17. Noborio-Hatano K, et al. Bortezomib overcomes cell-adhesion-mediated drug resistance through downregulation of VLA-4 expression in multiple myeloma. Oncogene. 2009;28(2):231-242.

18. Sripayap P, Nagai T, Hatano K, Kikuchi J, Furukawa Y, Ozawa K. Romidepsin overcomes cell adhesion-mediated drug resistance in multiple myeloma cells. Acta Haematol. 2014;132(1):1-4.

19. Isham CR, Tibodeau JD, Jin W, Xu R, Timm MM, Bible KC. Chaetocin: a promising new antimyeloma agent with in vitro and in vivo activity mediated via imposition of oxidative stress. Blood. 2007;109(6):2579-2588.

20. Xie ZG, et al. Determinants of sensitivity to DZNep induced apoptosis in multiple myeloma cells. PLoS One. 2011;6(6):e21583.

21. Zeng X, Chen S, Huang H. Phosphorylation of EZH2 by CDK1 and CDK2. Cell Cycle. 2011;10(4):579-583.

22. Yamaguchi H, Hung M-C. Regulation and role of EZH2 in cancer. Cancer Res Treat. 2014;46(3):209-222.

23. McMillin DW, et al. Tumor cell-specific bioluminescence platform to identify stroma-induced changes to anticancer drug activity. Nat Med. 2010;16(4):483-489.

24. Cha TL, et al. Akt-mediated phosphorylation of EZH2 suppresses methylation of lysine 27 in histone H3. Science. 2005;310(5746):306-310.

25. Chen S, et al. Cyclin-dependent kinases regulate epigenetic gene silencing through phosphorylation of EZH2. Nat Cell Biol. 2010;12(11):1108-1114.

26. Wei Y, et al. CDK1-dependent phosphorylation of EZH2 suppresses methylation of H3K27 and promotes osteogenic differentiation of human mesenchymal stem cells. Nat Cell Biol. 2011;13(1):87-94.

27. Harvey RD, Lonial S. PI3 kinase/AKT pathway as a therapeutic target in multiple myeloma. Future Oncol. 2007;3(6):639-647.

28. Liu P, et al. Cell-cycle-regulated activation of Akt kinase by phosphorylation at its carboxyl terminus. Nature. 2014;508(7497):541-545.

29. Tai YT, et al. Insulin-like growth factor-1 induces adhesion and migration in human multiple myeloma cells via activation of beta1-integrin and phosphatidylinositol 3'-kinase/AKT signaling. Cancer Res. 2003;63(18):5850-5858.

30. Kikuchi J, et al. Histone deacetylases are critical targets of bortezomib-induced cytotoxicity in multiple myeloma. Blood.2010;116(3):406-417.

31. Sasaki H, et al. Difference between genomic actions of estrogen versus raloxifene in human ovarian cancer cell lines. Oncogene. 2008;27(19):2737-2745.

32. Duan H, Heckman CA, Boxer LM. Histone deacetylase inhibitors down-regulate bcl-2 expression and induce apoptosis in $\mathrm{t}(14 ; 18) \mathrm{lym}$ phomas. Mol Cell Biol. 2005;25(5):1608-1619.

33. Shaffer AL, et al. IRF4 addiction in multiple myeloma. Nature. 2008;454(7201):226-231.
34. Chiron D, et al. Autocrine insulin-like growth factor 1 and stem cell factor but not interleukin 6 support self-renewal of human myeloma cells. Blood Cancer J. 2013;3:e120.

35. Hofgaard PO, et al. A novel mouse model for multiple myeloma (MOPC315.BM) that allows noninvasive spatiotemporal detection of osteolytic disease. PLoS One. 2012;7(12):e51892.

36 . Riedel SS, et al. Non-invasive imaging provides spatiotemporal information on disease progression and response to therapy in a murine model of multiple myeloma. PLoS One. 2012;7(12):e52398.

37. Schwarzer R, et al. Notch pathway inhibition controls myeloma bone disease in the murine MOPC315.BM model. Blood Cancer J. 2014;4:e217.

38. Rahal R, et al. Pharmacological and genomic profiling identifies NF-kB-targeted treatment strategies for mantle cell lymphoma. Nat Med. 2014;20(1):87-92.

39. Ernst $\mathrm{T}$, et al. Inactivating mutations of the histone methyltransferase gene EZH2 in myeloid disorders. Nat Genet. 2010;42(8):722-726.

40. Nikoloski G, et al. Somatic mutations of the histone methyltransferase gene EZH2 in myelodysplastic syndromes. Nat Genet. 2010;42(8):665-667.

41. Muto T, et al. Concurrent loss of Ezh2 and Tet2 cooperates in the pathogenesis of myelodysplastic disorders. J Exp Med. 2013;210(12):2627-2639.

42. Sashida G, et al. Ezh2 loss promotes development of myelodysplastic syndrome but attenuates its predisposition to leukaemic transformation. Nat Commun. 2014;5:4177.

43. Ntziachristos P, et al. Genetic inactivation of the polycomb repressive complex 2 in $\mathrm{T}$ cell acute lymphoblastic leukemia. Nat Med. 2012;18(2):298-301.

44. Simon C, et al. A key role for EZH2 and associated genes in mouse and human adult T-cell acute leukemia. Genes Dev. 2012;26(7):651-656.

45. Kim E, et al. Phosphorylation of EZH2 activates STAT3 signaling via STAT3 methylation and promotes tumorigenicity of glioblastoma stem-like cells. Cancer Cell. 2013;23(6):839-852.

46. Kawada H, et al. Rapid ex vivo expansion of human umbilical cord blood hematopoietic progenitors using a novel culture system. Exp Hematol. 1999;27(5):904-915.

47. Kikuchi J, et al. The novel orally active proteasome inhibitor K-7174 exerts anti-myeloma activity in vitro and in vivo by down-regulating the expression of class I histone deacetylases. J Biol Chem. 2013;288(35):25593-25602.

48. Kuhn DJ, et al. Targeting the insulin-like growth factor-1 receptor to overcome bortezomib resistance in preclinical models of multiple myeloma. Blood.2012;120(16):3260-3270.

49. Koyama D, et al. Proteasome inhibitors exert cytotoxicity and increase chemosensitivity via transcriptional repression of Notch1 in T-cell acute lymphoblastic leukemia. Leukemia. 2014;28(6):1216-1226. 